\title{
Shape optimisation of cold-formed steel columns with manufacturing constraints using the Hough transform
}

\author{
Bin Wang ${ }^{(1)^{\star}}$, Benoit P. Gilbert ${ }^{(1)}$, Adrien M. Molinier ${ }^{(1)}$, Hong Guan ${ }^{(1)}$ and Lip H. Teh ${ }^{(2)}$ \\ (1) Griffith School of Engineering, Gold Coast Campus, Griffith University, QLD 4222, Australia \\ (2) School of Civil, Mining and Environmental Engineering, University of Wollongong, NSW 2522, Australia \\ ${ }^{*}$ Corresponding author, Email: b.wang@griffith.edu.au
}

\begin{abstract}
This paper introduces manufacturing constraints into a recently developed evolutionary algorithm for shape optimisation of CFS profiles. The algorithm is referred to as "self-shape optimisation" and uses Genetic Algorithm (GA) together with the Augmented Lagrangian ( $A L$ ) method to avoid illconditioned problems. Simple manufacturing rules derived from the limitations of current coldforming processes, i.e. a limited ability to form continuously curved surfaces without discrete bends, are described in the paper and incorporated into the algorithm. The Hough transform is used to detect straight lines and transform arbitrarily drawn cross-sections into manufacturable ones. Firstly, the algorithm is verified against a known optimisation problem and found to accurately converge to a manufacturable optimum solution. Secondly, the algorithm is applied to singly-symmetric CFS columns each of which is subject to an axial compressive load of $75 \mathrm{kN}$ and has a uniform wall thickness of $1.2 \mathrm{~mm}$. The strength of the columns is evaluated by the Direct Strength Method (DSM) and all buckling modes are considered. Various column lengths (from $500 \mathrm{~mm}$ to $3,000 \mathrm{~mm}$ ) and numbers of roll-forming bends were investigated. The optimised cross-sections are presented and discussed.
\end{abstract}




\section{KEYWORDS}

Shape optimisation; Cold-formed steel structures; Hough transform; Genetic Algorithm. 


\section{INTRODUCTION}

Cold-formed steel (CFS) profiles are usually an attractive and cost-effective building structural solution relative to more "conventional" building materials, such as hot-rolled steel and concrete. They are thin-walled structural members with a high capacity-to-weight ratio [1] and can be prefabricated off-site and readily installed on-site.

The main advantage of CFS members lies in their manufacturing processes that allow the formation of almost any cross-sectional shape at room temperature. The profiles are typically formed by bending coils of thin steel sheets (up to $6 \mathrm{~mm}$ thick) with a finite number of rollers (roll-forming) or die blocks (brake-pressing). However, despite this flexibility, the use of CFS sections has been mainly restricted to Cee, Zed and Sigma cross-sectional shapes, with or without stiffeners, as shown in Fig. 1. As the cross-sectional shape controls the strength of CFS members, there is a real potential to eventually discover new optimised cross-sectional shapes tailored to specific applications, such as purlins, girts and studs for buildings, and uprights for storage racks for instance. Such discoveries will enhance the competitiveness of CFS structures and are now facilitated by the development of a new structural design method, the Direct Strength Method (DSM) [2], which allows any cross-sectional shapes with the same degree of complexity to be designed.

This paper aims at incorporating manufacturing constraints for optimising the cross-sectional shape of CFS columns by minimising the cross-sectional area for a given design axial compressive load. Optimisation for specific applications is not considered in the present study, and will be investigated in the future. Simple roll-forming (or brake-pressing) manufacturing rules are defined and incorporated into the recently developed "self-shape optimisation" algorithm $[3,4]$ as manufacturing constraints. The specificity of the algorithm is briefly described in the paper. The Hough transform, used to detect straight lines and transform non-manufacturable cross-sections into manufacturable ones, is explained herein. The algorithm is verified against a known optimisation problem, being the optimisation of the cross-sectional shape of a doubly-symmetric closed thin-walled profile for given 
second moments of area. The verified algorithm is then used to optimise simply-supported, singlysymmetric and free-to-warp open-section CFS columns. The optimised manufacturable crosssections are presented and discussed in the paper. For comparison purposes, the algorithm is also run to obtain non-manufacturable cross-sections.

\section{BACKGROUND}

\subsection{General}

One of the early studies on shape optimisation of CFS profiles is attributed to Liu et. al. [5]. A knowledge-based global optimisation algorithm, aiming at optimising the capacity of CFS columns manufactured from coils of set width and thickness, was used. Leng et. al. [6] optimised the crosssectional shapes of CFS open columns using three different optimisation algorithms, namely gradient-based steepest descent method, Genetic Algorithm (GA) and Simulated Annealing (SA). Sections having a wall thickness of $1 \mathrm{~mm}$ and a perimeter of $280 \mathrm{~mm}$ were divided into 21 elements and optimised. "Open circular" and "S" cross-sections were found. Moharrami et. al. [7] improved the study in [6] by introducing various types of boundary conditions into the algorithm. Gilbert et. al. [3] proposed a GA-based Augmented Lagrangian ( $A L$ ) constraint-handling shape optimisation method for CFS profiles. The accuracy of the algorithm was verified against an optimisation problem for which an analytical solution is known. Gilbert et. al. [4] then applied the verified algorithm to optimise the cross-sectional shape of CFS simply-supported, singly-symmetric and open-section columns, subjected to a certain axial compressive load. A set of rules to accurately determine the local and distortional elastic buckling stresses from the Finite Strip signature curves was also developed.

Manufacturing constraints were first introduced into shape optimisation algorithms for CFS profiles by Leng et. al. [8]. The authors introduced partial manufacturing and construction (geometric end uses) constraints using an SA algorithm. The constraints were implemented by defining (i) flat 
"horizontal" flanges, (ii) minimum dimensions for the "vertical" web, flanges and lips, (iii) allowance passage for utilities between lips and (iv) no overlapping elements in the cross-sections. The study was improved in Leng et. al. [9] by introducing a limited number of rollers (representing the number of discrete bends between flat segments, see Section 2.3). These enhancements resulted in manufacturable cross-sections with improved capacities when compared to conventional Ceesections of identical cross-sectional area. Leng et. al. [10] also presented optimised cross-sectional shapes, i.e. singly-symmetric "Cee" and "Sigma" and anti-symmetric "S", with both manufacturing and construction constraints. Franco et. al. [11] proposed CFS shape grammar rules, with an "alphabet", for shape optimisation of CFS profiles. Manufacturing constraints, with given stiffener sizes, were intrinsic to the shape grammar resulting in manufacturable cross-sections. Genetic Algorithm (GA) was used in [11] as a search algorithm.

\subsection{Present shape optimisation algorithm}

The algorithm referred to as "self-shape optimisation" and developed in $[3,4]$ is used in the current study. The method rigorously explores the natural evolution process and the latent potential of GA in an innovative way. GA was initially developed by Holland [12] and is an adaptive heuristic search algorithm that mimics the Darwin's evolutionary survival of the fittest theory. It is less susceptible to be self-trapped into local optima, and is able to handle non-linear problems. The classical GA principles can be found in Adeli and Sarma [13].

GA is an unconstrained optimisation method, and constrained problems are transformed into unconstrained problems by using a fitness function $f$ expressed as,

$$
f=f(x)+\sum_{i=1}^{n} \alpha_{i} g_{i}(x)+\sum_{i=n+1}^{k} \beta_{i} h_{i}(x)
$$


where $f(x)$ is the objective function, $x$ is the vector of design variables, $g_{i}(x)$ and $h_{i}(x)$ are the $t^{\text {th }}$ inequality and equality constraint violations ( $n$ inequality and $k-n$ equality constraints), respectively, and $\alpha_{i}$ and $\beta_{i}$ are penalty factors. The algorithm aims at minimising $f$.

In theory, the penalty factors in Eq. (1) increase when the constraint violations decrease and convergence can be facilitated by increasing the penalty factors. Nevertheless, large values of the penalty factors lead to ill-conditioned problems or slow down the algorithm [14, 15]. To avoid the problem of having penalty factors increasing to infinity, the Augmented Lagrangian $(A L)$ constrainthandling method for GA proposed by Adeli and Cheng [14] is used in this research to solve the problem. The fitness function $f$ is then re-arranged as,

$$
f=f(x)+\frac{1}{2}\left\{\sum_{i=1}^{n} \gamma_{i}\left\{\max \left[0, g_{i}(x)+\mu_{i}\right]\right\}^{2}+\sum_{i=n+1}^{k} y_{i}\left[h_{i}(x)+\mu_{i}\right]^{2}\right\}
$$

where $\gamma_{i}$ and $\mu_{i}$ are penalty function coefficients and real parameters associated with the $i^{\text {th }}$ inequality and equality constraints, respectively. $\gamma_{i}$ and $\mu_{i}$ are automatically updated at each GA generation but are kept to finite values [14].

The main characteristics of the "self-shape optimisation" principle [3] are summarised below:

- The initial population in the GA is generated by arbitrarily drawing cross-sections using selfavoiding random walks in a defined design space. These random walks enable cross-sections to be generated without presumptions of their shapes.

- A floating-point type GA is used, implying that a cross-section is defined by floating-point numbers representing the coordinates of the points constituting the cross-section.

- Cross-over and mutation operators are performed in relation to the design space but not to the floating-point variables. The cross-over operator allows for the merging of two cross-sections to generate off-springs bearing similarity in cross-sectional shapes to the two parents. In the mutation operator, a part of the cross-section is deleted and redrawn. 


\subsection{Manufacturing constraints}

\subsubsection{Traditional manufacturing processes}

CFS profiles are typically mass-produced by two main cold-forming processes, referred to as "rollforming" and "brake-pressing". Both processes involve bending a flat sheet of steel to a desired cross-sectional shape. In roll-forming operations, as shown in Fig. 2 (a), the sheet is gradually rolled to a desired cross-sectional shape through successive rollers. This continuous manufacturing process allows long profiles to be manufactured. In brake-pressing operations, as shown in Fig. 2 (b), the sheet is repetitively pressed between differently shaped brake punches and die blocks to bend it to the desired cross-sectional shape. Brake-pressing is limited in manufacturing long members. Both manufacturing processes can only bend the flat sheet of metal at discrete bending locations, leaving flat (straight) segments between bends. This limitation needs to be considered in the shape optimisation algorithms to obtain manufacturable cross-sections.

\subsubsection{Simple manufacturing rules}

Simple manufacturing rules have been defined herein based on the basic roll-forming constraints encountered by a European steel storage rack manufacturer. They consist of three main rules:

(1) The minimum internal bending radius $r$ to steel sheet thickness $t$ ratio is 1.0 ;

(2) The minimum length of a single flat segment is $10 \mathrm{~mm}$;

(3) The number of flat segments per cross-section cannot exceed 20 (i.e. a maximum number of 19 bends per open cross-section and 10 flat segments per half cross-section).

In the present study, Rule (1) is neglected since it does not affect the basic shape of the optimised manufacturable cross-section, and only Rules (2) and (3) are considered. A nil internal bending 
radius (i.e. perfect bends) is assumed to simplify the algorithm. Actual bending radii can be added to the optimised cross-section prior to manufacture.

\subsubsection{Hough transform}

The Hough transform is used in this paper to detect straight lines, i.e. flat manufacturable segments, in the cross-section. This transform is commonly used in image processing to detect regular shapes, such as straight lines, circles and ellipses, from the discrete points forming the image [16].

The method consists of defining a "parametric space" in which each straight line in the image is represented by its orientation angle $\theta$, with respect to the Cartesian $x$-axis, and its normal distance $r$ to the origin, as shown in Fig. 4(a). If $\theta$ is restricted to the interval $\left[0^{\circ} ; 180^{\circ}\right.$, each straight line is represented by an unique coordinate $(r, \theta)$ in the parametric space. An image point of coordinate $\left(x_{i}\right.$, $\left.y_{i}\right)$ in the Cartesian $x-y$ space is transformed into a sinusoidal curve in the parametric $r-\theta$ space as

$$
r=x_{i} \cos \theta+y_{i} \sin \theta
$$

Sinusoidal curves having common intersecting points have collinear (aligned) points in the image. This is illustrated in Fig. $4(\mathrm{~b})$ with 4 points aligned on the line of coordinate $\left(r=10 \mathrm{~mm}, \theta=60^{\circ}\right)$ in the parametric space.

For image processing purposes, an array referred to as the accumulator array (or accumulator matrix), is created in the discretised parametric space. The columns of the array correspond to the increasing values of $\theta$, at $\Delta \theta$ intervals, and the lines to increasing values of $r$, at $\Delta r$ intervals. Aligned image points are detected as,

- Step 1: Set $\theta=0^{\circ}$. 
- Step 2: For each image point (i) calculate its $r$ value from Eq. (3) for the set value of $\theta$, (ii) calculate the closest discrete $r$ value matching the lines of the accumulator array and (iii) add the point reference number to the corresponding cell in the accumulator array.

- Step 3: Set $\theta=\theta+\Delta \theta$. If $\theta \geq 180^{\circ}$ go to Step 4, else go to Step 2 .

- Step 4: All points sharing the same cell in the accumulator array are considered aligned.

The choice of the intervals $\Delta \theta$ and $\Delta r$ influences the ability and accuracy of the Hough transform in detecting straight lines. The smaller $\Delta \theta$, the more refined the search space. A larger value of $\Delta r$ (wider corridor) represents a less stringent alignment tolerance, as illustrated in Fig. 5, where two values of $\Delta r$ are shown. A larger $\Delta r_{1}$ results in all the four points in Fig. 5 being aligned by the Hough transform. A smaller $\Delta r_{2}$ results in only two points being aligned by the Hough transform.

\subsubsection{Manufacturing constraints in the GA}

The manufacturing constraints are introduced into the fitness function (see Eq. (1)) as an equality constraint $h_{\text {align, expressed as, }}$

$$
h_{\text {align }}=\omega\left|\frac{\text { nbAligned }}{\text { nbElement }}-1\right|
$$

where $\omega$ is a weight associated with the constraint, nbElement is the total number of elements per half cross-section and nbAligned represents the number of aligned elements composed of the longest non-concurrent flat segments per half cross-section. The number of non-concurrent flat segments is taken to $N_{\max }$ if there are more than $N_{\max }$ non-concurrent flat segments per half crosssection or to the actual number of non-concurrent flat segments otherwise. $N_{\max }$ corresponds to a maximum number of flat segments per half cross-section set by the manufacturer, with $N_{\max }$ less than or equal to the maximum possible number of flat segments defined in Rule (3) outlined in Section 2.3.2. In the algorithm, a flat segment is determined from the Hough transform as 
consecutive aligned cross-sectional elements of total length equal to or greater than the minimum manufacturable length as defined in Rule (2) in Section 2.3.2. If the cross-section is made of less than $N_{\max }$ flat segments, the constraint is considered satisfied and $h_{\text {align }}=0$.

\section{VALIDATION}

\subsection{Optimisation problem}

A similar optimisation problem to the one used in [3], for which an analytical solution exists, is used herein to verify the ability and accuracy of the algorithm in optimising manufacturable crosssectional columns. It consists of minimising the cross-sectional area $A_{s}$ of a thin-walled bisymmetric closed cross-section for given second moments of area, $I_{x t}$ and $I_{y t}$, about the two axes of symmetry. Ragnedda and Serra [17] indicated that, when $I_{x t}$ equals $I_{y t}$, the optimised cross-section is a circle and therefore a regular polygon of $n$ sides if the cross-section is manufactured with $n$ flat segments.

A regular octagon $(n=8)$ with apothem a (at mid-wall thickness) of $20 \mathrm{~mm}$ and wall thickness $t$ of 1 $\mathrm{mm}$ is used herein to verify the algorithm. The cross-sectional area of the octagon $A_{o}$ is $132.55 \mathrm{~mm}^{2}$ and the length $L_{\text {side }}$ of one side for the octagon is therefore $16.6 \mathrm{~mm}$. Its second moments of area $I_{x t}$ $=I_{y t}$ are $28043.3 \mathrm{~mm}^{4}$. As the problem is bisymmetric, only a quarter of the cross-section in Fig. 6 is optimised herein and the maximum number of flat segments $N_{\max }$ is therefore set to 2 . The fitness function $f$ derived from Eqs. (1) and (4) is expressed as,

$$
\text { Minimise } f=\frac{A_{s}}{A_{o}}+\alpha_{x} \max \left(0,1-\frac{I_{x}}{I_{x t}}\right)+\alpha_{y} \max \left(0,1-\frac{l_{y}}{l_{y t}}\right)+\alpha_{\text {align }} \omega\left|\frac{n b \text { Aligned }}{n b E l e m e n t}-1\right|
$$

where $I_{x}$ and $I_{y}$ are the calculated second moments of area of the cross-section, and $\alpha_{x}, \alpha_{y}$ and $\alpha_{a l i g n}$ are penalty factors associated with each constraint. In Eq. (5), the constraints on the given second 
moments of area are expressed as inequality constraints. In other words, the algorithm is not penalised if $I_{x} \geq I_{x t}$ or $I_{y} \geq I_{y t}$. This was found to have significantly improved convergence.

In Fig. 6, the circle with the same second moment of area and wall thickness as the octagon is also shown for comparison. The cross-sectional area $A_{c}$ of the circle is $130.31 \mathrm{~mm}^{2}$, i.e. $1.7 \%$ less than that of the manufacturable octagon.

The AL fitness function used in the algorithm and derived from Eqs. (2) and (5) is given as,

$$
f=\frac{A_{s}}{A_{o}}+\frac{1}{2}\left\{\gamma_{x}\left[\max \left(0,1-\frac{I_{x}}{I_{x t}}+\mu_{x}\right)\right]^{2}+\gamma_{y}\left[\max \left(0,1-\frac{I_{y}}{I_{y t}}+\mu_{y}\right)\right]^{2}+\gamma_{\text {align }}\left[\omega\left|\frac{n b \text { Aligned }}{n b E \text { lement }}-1\right|+\mu_{\text {align }}\right]^{2}\right\}
$$

where $\gamma_{x}, \gamma_{y}$ and $\gamma_{a l i g n}$ are the $\mathrm{AL}$ penalty function coefficients, $\mu_{x}, \mu_{y}$ and $\mu_{\text {align }}$ are the real parameters associated with each penalty function coefficient. Gilbert et al. [3] investigated the appropriate initial values for $\gamma_{x}$ and $\gamma_{y}$, and their recommendation of $\gamma_{x}=\gamma_{y}=2.0$ is used herein. For initial values of $\gamma_{x}$ and $\gamma_{y}$ less than 0.5 , the algorithm tends to select cross-sections with a small number of elements as the fittest ones. The appropriate initial value of $V_{\text {align }}$, associated with $\omega$, is investigated in Section 3.3.1. Initial values of $\mu_{x}=\mu_{y}=\mu_{\text {align }}=0$ are used, as recommended by Belegundu and Arorat [18].

\subsection{Other parameters used}

An AL penalty increasing constant $\beta$ of 1.05 and a convergence rate $\rho$ of 1.5 , as recommended in [3], are used. A value of $\beta$ greater than 1.5 forces the algorithm to converge prematurely [3]. The design space is set to $40 \mathrm{~mm} \times 40 \mathrm{~mm}$ [3] and the maximum number of generations to 150 per run. Ten runs are performed to verify the robustness of the algorithm. The number of individuals is set to 700 per generation and the cross-sections are drawn with short elements of nominal length of $2 \mathrm{~mm}$ 
(see [3] for more details). The probabilities of cross-over and mutation operations in the GA are equal to $80 \%$ and $1 \%$, respectively, as used in [3].

\subsection{Parametric studies and results}

\subsubsection{AL penalty function coefficient Yalign and associated weight $\omega$}

In order to determine the appropriate initial value for Yalign and constant value for weight $\omega$, nine different combinations are investigated, as shown in Table 1. The value of $Y_{\text {align }}$ and $\omega$ are selected in the intervals $[0,1]$. The alignment tolerance and step of orientation angle in the Hough transform are set to $\Delta r=0.5 t$ and $\Delta \theta=0.5^{\circ}$, respectively, in this section.

Fig. 7 illustrates the average fitness function $f$ given in Eq. (5) over 10 runs, with $\alpha=10$, for all studied combinations of $Y_{\text {align }}$ and $\omega$. The convergence rate improves as $Y_{\text {align }}$ increases from 0.01 to 1.0. The algorithm tends not to converge when $Y_{\text {align }}$ is too small (equal to 0.01 ), as the weight of the alignment penalty function in the AL fitness function in Eq. (6) is too small when compared to the objective function. The algorithm, on the other hand, is able to converge to a solution for the remaining studied combinations (4) to (9).

Table 2 compares the average results over 10 runs to the known optimum solution for all studied combinations. A negative sign in Table 2 means that the optimised results are less than the optimum solution. Combination (1), i.e. $Y$ align $=0.01$ and $\omega=0.3$, experiences incomplete alignment with a relative alignment error of $-1.1 \%$, while all the remaining combinations allow complete alignment. The average second moments of area are similar to the optimum ones for all combinations within a maximum average error of $1.4 \%$ (combination (8)). Yet, the coefficient of variation $(\mathrm{CoV})$ on the second moments of area is minimum for Valign $=0.1$ (combination (4) to (6)). The minimum average cross-sectional area of $131.96 \mathrm{~mm}^{2}$ is found for combination (5) and is $0.4 \%$ less than the one of the optimum octagon, therefore implying this combination represents a more 
optimal solution. Combination (5), i.e. Yalign $=0.1$ and $\omega=0.5$, is therefore chosen for further analysis in this study.

\subsubsection{Alignment tolerance $\Delta r$}

Table 3 shows the values of $\Delta r$ and corresponding alignment tolerance ratios $\Delta r / L_{\text {side }}$ analysed to find the appropriate value of the alignment tolerance to ensure convergence and accuracy of the algorithm. $\Delta \theta$ is kept constant at $0.5^{\circ}$.

Similar to Fig. 7, Fig. 8 plots the average fitness function $f$ over 10 runs for all studied cases of $\Delta r$. The algorithm always converges to a solution. The larger the alignment tolerance $\Delta r$, the faster the convergence rate is, with $\Delta r=2.0 t$ converging at the $50^{\text {th }}$ generation approximately. The algorithm converges in about 100 generations for $\Delta r=0.5 t$.

Table 4 summarises the average results for all studied cases of $\Delta r$. The algorithm always satisfies the alignment criteria and converges to consistent solutions with small CoVs (within $0.5 \%$ ) for all cases. In the case $\Delta r=2.0 t$, the average relative errors on the cross-sectional area and second moments of area about the two axes of symmetry are $-1.6 \%(\mathrm{CoV}=0.0 \%)$ and $0.0 \%(\mathrm{CoV}=0.1 \%)$, respectively. This average cross-sectional area $\left(130.43 \mathrm{~mm}^{2}\right)$ is closer to (less than $\left.0.1 \%\right)$ the absolute optimum circle cross-sectional area than the targeted octagon. To illustrate, Fig. 9 plots the fittest optimised cross-sections, with the wall thickness of $1 \mathrm{~mm}$ shown, at the $150^{\text {th }}$ (final) generation for all cases of $\Delta r$. The larger the alignment tolerance is, the closer the cross-section to the absolute optimum circle is. A "circle" like shape is mainly observed for the case $\Delta r=2.0 t$, while an "octagon" like shape is mainly observed for the other cases. Fig. 10 shows the second, third, second least and least fit optimised cross-sections, out of ten runs, for the stringiest alignment tolerance, $\Delta r=0.5 t$. The fittest cross-section for this case is given in Fig. 9 (a). All cross-sections can converge to a consistent "octagon" shape outlining the robustness of the algorithm. 
Based on the above results and as a simple rule, an alignment tolerance ratio $\Delta r / L_{\text {side }}$ greater than 0.03 but no more than 0.1 is used in the Hough transform through this research, as a compromise between accuracy and convergence rate. Alignment tolerance ratios $\Delta r / L_{\text {side }}$ less than 0.03 would allow more optimal shapes, i.e. closer to the octagon, but will require a large number of generations to be analysed and may cause convergence issues due to a stringent alignment tolerance.

\section{OPTIMISATION OF CFS COLUMNS}

\subsection{Optimisation problem}

The validated algorithm is applied to minimise the cross-sectional area $A_{s}$ of manufacturable CFS columns subjected to an axial compressive force $N^{*}$ of $75 \mathrm{kN}$. The columns are simply-supported, symmetric and free to warp, with a uniform wall thickness $t$ of $1.2 \mathrm{~mm}$. Column lengths of $500 \mathrm{~mm}$ (short), 1,500 mm (intermediate) and 3,000 $\mathrm{mm}$ (long) are investigated. The yield stress $f_{y}$ of the column is $450 \mathrm{MPa}$, the Young's modulus $E$ is $200 \mathrm{GPa}$ and the shear modulus $\mathrm{G}$ is $80 \mathrm{GPa}$. The optimisation problem is illustrated in Fig. 11. The algorithm is also run without considering the manufacturing constraints for comparison purposes.

The constrained optimisation problem is transformed into an unconstrained problem suitable for the GA and involves minimising the fitness function $f$,

$$
f=\frac{A_{s}}{A_{\text {squash }}}+\alpha \max \left(0, \frac{N^{*}}{N_{c}}-1\right)+\alpha_{\text {align }} \omega\left|\frac{n b \text { Aligned }}{\text { nbElement }}-1\right|
$$

where the first term in the equation represents the objective. $A_{\text {squash }}$ is the squash area, defined as the lower bound cross-sectional area of the profile: 


$$
A_{\text {squash }}=\frac{N^{*}}{f_{y}}
$$

The second term in Eq. (7) represents the constraint on the axial capacity and is expressed as an inequality constraint. In other words, the cross-section is not penalised if its capacity exceeds the targeted capacity of $75 \mathrm{kN} . N_{c}$ is the nominal member capacity in compression, calculated from the Australian standard AS4600 [19] (see Section 3.1), and $\alpha$ is the penalty factor associated with the capacity constraint. The last term represents the manufacturing constraint and is expressed as an equality constraint (see Section 2.3.4). $N_{\max }$ is the maximum possible number of flat segments per half cross-section set by the manufacturer, but is less than the upper limit of flat segments defined in Rule (3) in Section 2.3.2. In the algorithm, a flat segment is determined from the Hough transform as consecutive aligned cross-sectional elements of a total length equal to or greater than the minimum manufacturable length $(10 \mathrm{~mm})$, defined in Rule (2) in Section 2.3.2. If the half cross-section is made of less than $N_{\max }$ flat segments, the constraint is considered satisfied.

The AL method for GA proposed by Adeli and Cheng [14] is also used herein to handle the axial capacity and manufacturing constraints. The fitness function $f$ is then expressed as,

$$
f=\frac{A_{s}}{A_{\text {squash }}}+\frac{1}{2}\left\{\gamma\left[\max \left(0, \frac{N^{*}}{N_{c}}-1+\mu\right)\right]^{2}+\gamma_{\text {align }}\left[\omega\left|\frac{n b \text { Aligned }}{n b \text { Element }}-1\right|+\mu_{\text {align }}\right]^{2}\right\}
$$

where $\gamma$ and $V$ align are the penalty function coefficients, and $\mu$ and $\mu_{\text {align }}$ are the real parameters associated with each penalty function coefficient. From the parametric study in Section 3 and [3], initial values of $\gamma=2.0, V_{\text {align }}=0.1, \mu=\mu_{\text {align }}=0$ and $\omega=0.5$ are used. An AL penalty increasing constant $\beta$ of 1.05 and a convergence rate $\rho$ of 1.5 are also used [3].

\subsection{Column capacity}




\subsubsection{Design method for CFS columns}

Similar to [4], the DSM [2] as detailed in Section 7 of the Australian Standard AS4600 [19] is used in this research to calculate the nominal member capacity in compression, $N_{c}$ of the cross-sections given as,

$$
N_{c}=\min \left(N_{c e}, N_{c l}, N_{c d}\right)
$$

where $N_{c e}, N_{c l}$ and $N_{c d}$ are the nominal axial compressive capacities for global, local and distortional buckling, respectively (see [19] for more details).

\subsubsection{Determination of the elastic buckling stresses}

In this study, the elastic global buckling stress $f_{o c}$ of the cross-sections is estimated from Timoshenko's buckling theory, as given in Clause 3.4.3 for singly-symmetric open cross-section of the Australian Standard AS4600 [19]. The rules developed in [4] to automatically estimate the elastic local and distortional buckling stresses $f_{o l}$ and $f_{o d}$, respectively, from the Finite Strip Method (FSM) [20] and the constrained Finite Strip Method (cFSM) [21] signature curves, are used. The open source software CUFSM [22] is used to perform the Finite Strip analyses.

\subsection{Other parameters}

To study the influence of the maximum number of discrete bends on the optimised cross-sectional shape, various maximum numbers of flat segments $N_{\max }$ per half cross-section are investigated. The number of investigated flat segments $N_{\max }$ considered for each column length is given in Table 5. $\Delta r$ is adjusted to the value of $N_{\max }$ and column lengths according to the results obtained from Section 3.3.2. Specifically, the maximum length of the cross-sectional elements $L_{\max }$ for the optimised 
solutions was first estimated for each combination of $N_{\max }$ and column length by pre-running the algorithm with a large alignment tolerance. $\Delta r$ was then chosen for each case to satisfy $\Delta r / L_{\max }$ within the interval $[0.03,0.1]$. The value of $\Delta r$ for each studied case is given in Table 5. The step of orientation angle $\Delta \theta$ is set to $1^{\circ}$ for all cases.

In view of the parameters of the GA, the design space is set to $100 \mathrm{~mm} \times 100 \mathrm{~mm}$ [3] and the maximum number of generations per run to 150 for manufacturable cross-sections and 80 [4] for non-manufacturable ones. 10 runs are performed. The number of individuals per generation is set to 500 and the cross-sections are arbitrarily drawn with short elements of nominal length of $4 \mathrm{~mm}$ [3]. The cross-over and mutation operators for singly-symmetric cross-sections detailed in [4] apply. The probabilities of the cross-over and mutation operators in the GA are equal to $80 \%$ and $1 \%$, respectively [3].

To improve computation time when elements are aligned, i.e. forming a flat segment, only the coordinates of the flat segments are entered into CUFSM [22]. However, for accuracy of the Finite Strip analysis [10], flat segments between $10 \mathrm{~mm}$ to $15 \mathrm{~mm}$ in length are divided into two segments of equal length, and flat segments longer than $15 \mathrm{~mm}$ are divided into three segments of equal length.

A simplified flowchart is presented in Fig. 12 showing that half cross-sections are used in the GA and entire cross-sections are only formed for elastic bucking analyses in CUFSM [22]. The detailed flowchart of the algorithm is shown in [3]. While singly-symmetric and/or anti-symmetric sections can be readily incorporated into the algorithm, only singly-symmetric columns are optimised in this study.

\subsection{Results and discussion}




\subsubsection{Convergence}

Fig. 13 shows the average fitness functions $f$ given in Eq. (7) times the squash area $A_{\text {squash }}$ over 10 runs for 500 mm (Fig. 13(a)), 1,500 mm (Fig. 13(b)) and 3,000 mm (Fig. 13(c)) long columns. In Fig. 13, the penalty factor in Eq. (7) is set to $\alpha=10$. The algorithm always converges to an optimised solution. Typically, the higher $N_{\max }$, the fastest the convergence is, likely because the length of the flat segments of the optimised cross-sections increases when $N_{\max }$ decreases. The algorithm always converges the fastest for the non-manufacturable cases. The algorithm approaches an optimised solution at about the $100^{\text {th }}$ generation for all manufacturable cases of the $500 \mathrm{~mm}$ long columns (Fig. 13(a)), $N_{\max }=5$ to 7 of the $1,500 \mathrm{~mm}$ long columns (Fig. 13(b)) and $N_{\max }=5$ to 8 of the 3,000 $\mathrm{mm}$ long columns (Fig. 13(c)). An optimum solution is approached after the $140^{\text {th }}$ generation for the remaining cases. For the non-manufacturable cases, 60 generations are sufficient to approach an optimised solution for all column lengths.

The computation time is highly dependent on the number of cross-sectional elements analysed in CUFSM [22] (written in MATLAB), to perform the Finite Strip analyses. The greater the number of elements, the longer the computation time is. The main algorithm is written in Python and does not use parallel processing, while the Finite Strip analyses operated in CUFSM does. Up to 8 computer cores are selected per MATLAB analysis in a high performance computer cluster consisting of a mixture of SGI Altix XE and SGI ${ }^{\circledR}$ Rackable $^{\mathrm{TM}}$ C2114-4TY14 servers for this purpose. The Finite Strip analyses take about $80 \%$ of the computation time and the main algorithm takes the remaining $20 \%$. It takes on average 20,35 and 65 minutes to optimise one generation for the manufacturable columns and 30, 50 and 75 minutes for the non-manufacturable ones for the $500 \mathrm{~mm}, 1,500 \mathrm{~mm}$ and 3,000 mm long columns, respectively.

In this study, a strict maximum of 75,000 solutions are evaluated (150 generations $\times 500$ crosssections) per run with the algorithm often converging in less than 50,000 solutions. This number is of the same order of magnitude as the 40,000 solutions evaluated in $[6,23]$. 


\subsubsection{Average results}

Table 6 to Table 8 summarise the average results over 10 runs for the $500 \mathrm{~mm}, 1,500 \mathrm{~mm}$ and $3,000 \mathrm{~mm}$ long columns, respectively. When the algorithm is run with manufacturing constraints, the algorithm always satisfies the alignment criteria and converges to consistent solutions with small CoVs on the cross-sectional area (maximum of $0.91 \%$ when $N_{\max }=3$ in Table 7 ). This further confirms the robustness of the algorithm. The average nominal member capacity $N_{c}$ is always close to the targeted axial compressive capacity of $75 \mathrm{kN}$, with the largest average absolute error equal to $0.50 \%$ for the $3,000 \mathrm{~mm}$ long columns when $N_{\max }=3$ (see Table 8). For all cases, except for $N_{\max }=$ 7 and 8 of the $3,000 \mathrm{~mm}$ long columns, the average ultimate compressive stress (nominal axial capacity-to-area ratio) is always lower than the same of the non-manufacturable solutions, as the latter are more optimum. However, for $N_{\max }=7$ and 8 of the $3,000 \mathrm{~mm}$ long columns (Table 8 ), the average ultimate compressive stress is up to $0.6 \%$ higher than the same of the non-manufacturable cross-sections. This outcome is due to the extremely close cross-sectional shapes between the manufacturable and non-manufacturable cases and the large number of elements for the nonmanufacturable cross-sections that limits the ability of the algorithm in forming perfectly curved cross-sections. The lowest average ultimate compressive stress of the manufacturable cases, found for $N_{\max }=3$, is $1.1 \%, 2.5 \%$ and $1.9 \%$ lower than the same of the non-manufacturable case for the $500 \mathrm{~mm}$ (Table 6), 1,500 mm (Table 7) and 3,000 mm (Table 8) long columns, respectively. This validates the finding in [8] that introducing manufacturing constraints into shape optimisation algorithms marginally reduces the performance of the sections.

\subsubsection{Cross-sectional shapes}

Fig. 14 to Fig. 18 illustrate the optimised non-manufacturable (Fig. 14) and manufacturable (Fig. 15 to Fig. 18) cross-sections for the $500 \mathrm{~mm}$ long columns. Based on the value of the ultimate compressive stress, the two fittest and two least fit cross-sections are shown in each figure. The two 
fittest cross-sections for all cases typically converge to "bean" cross-sectional shapes (subscripts (ab) in Fig. 14 to Fig. 18), while the least fit ones mostly converge to open "Cee" sections (Fig 13 (c), Fig. 16 (d), Fig. 17 (d) and Fig. 18 (c, d)). Fig. 17 (c) shows a closed "Cee" section, Fig. 14 (d) and Fig. 15 (d) a "Sigma" cross-sectional shape, and Fig. 14 (c) and Fig. 16 (c) a "bean" cross-sectional shape. The fittest non-manufacturable cross-section in Fig. 14 (a) has the largest ultimate compressive stress, $387.8 \mathrm{MPa}$, of all cross-sections. Its cross-sectional depth and width are 64.9 $\mathrm{mm}$ and $36.5 \mathrm{~mm}$, respectively, i.e. a depth-to-width ratio of 1.78 . The case $N_{\max }=3$ (Fig. 15 (a)) has the lowest ultimate compressive stress of all fittest manufacturable cross-sections, which is only $0.9 \%$ lower than that of the fittest non-manufacturable solution (Fig. 14 (a)). Its cross-sectional depth and width are $5.2 \%$ and $1.4 \%$, respectively, greater than the fittest non-manufacturable solution.

Similar to Fig. 14 to Fig. 18, Fig. 19 to Fig. 24 show the fittest and least fit optimised cross-sections for the 1,500 mm long columns. The fittest cross-sections converge to "bean" (Fig. 19 (a, b), Fig. 20 (a, b), Fig. 22 (b), Fig. 23 (b) and Fig. 24 (a, b)) and closed "Cee" (Fig. 21 (a, b), Fig. 22 (a) and Fig. 23 (a)) cross-sectional shapes, while the least fit ones converge to "Sigma" cross-section shapes (subscripts (c, d) in Fig. 19 and Fig. 21 to Fig. 23) and nearly closed "Cee" (Fig. 20 (c, d)). For $N_{\max }$ = 7, Fig. 24 (c, d) show closed (or nearly closed) "Cee" sections with lip stiffeners.

Similar conclusions to the ones drawn for the $500 \mathrm{~mm}$ long columns apply: (i) the fittest nonmanufacturable cross-section (Fig. 19 (a)) has the largest ultimate compressive stress (261.0 MPa) of all studied cases, and (ii) the fittest manufacturable cross-section with the lowest ultimate compressive stress $\left(N_{\max }=3\right.$ in Fig. 20 (a)) performs similarly to the fittest non-manufacturable solution, with only $2.5 \%$ difference in ultimate compressive stresses between the two solutions. The cross-sectional depth and width of the former manufacturable cross-section are $7.5 \%$ and $2.8 \%$, respectively, lower than that of the fittest non-manufacturable solution.

Fig. 25 to Fig. 31 show the two fittest and two least fit optimised non-manufacturable and manufacturable cross-sections for the $3,000 \mathrm{~mm}$ long columns. All cross-sections converge to 
"bean" cross-sectional shapes, with the least fit cross-sections usually having a more open crosssectional shape than the fittest ones. The large number of elements forming the non-manufacturable cross-sections in Fig. 25 results in not perfectly curved shapes, and the largest ultimate compressive stress (173.6 MPa) is found for the manufacturable case $N_{\max }=6$ in Fig. 29 (a). However, this value is only $0.11 \%$ greater than the ultimate compressive stress of the fittest nonmanufacturable case in Fig. 25 (a). The cross-sectional depth and width of the fittest cross-section are $134.1 \mathrm{~mm}$ and $82.6 \mathrm{~mm}$, respectively, which represents a cross-section that is $1.8 \%$ less deep and $4.2 \%$ wider than the fittest non-manufacturable solution. The ultimate compressive stresses of the fittest cross-sections for all studied cases are close to each other and no more than $1.8 \%$ apart.

\subsubsection{Improvement in capacity}

The nominal member capacities in compression of the optimised manufacturable solutions in Table 6 through Table 8 are compared to conventional lipped channel cross-sections that have similar aspect ratios to the sections manufactured in Australia by Bluescope Lysaght [24] and the same cross-sectional area of the optimised sections. Table 9 summarises the dimensions and capacity of the conventional lipped channels used for each investigated column length. The conventional sections in Table 9 satisfy the geometric limitations for pre-qualified DSM compression members given in Table 7.1.1 of Australian standard AS4600 [19]. As noted in Table 9, the nominal member capacities of the optimised solutions are significantly larger than the corresponding conventional ones. The improvement ranges from $30 \%$ for the $500 \mathrm{~mm}$ long columns to $151 \%$ for the $3,000 \mathrm{~mm}$ long columns.

\section{CONCLUSIONS}

This paper has defined a set of simple manufacturing rules and incorporated them into the previously developed "self-shape optimisation" algorithm for CFS profiles using the Hough transform. The objective of the fitness function is to minimise cross-sectional area subjected to a targeted axial 
member compressive capacity and manufacturing constraints. The ability and accuracy of the algorithm in optimising manufacturable thin-walled cross-sections have been verified against a known optimisation problem. The algorithm was used to shape-optimise manufacturable simplysupported, singly-symmetric and free to warp CFS open-section columns for all buckling modes. Short, intermediate and long columns, with various numbers of manufacturable flat segments, were investigated. The main conclusions are summarised below:

- The robustness of the algorithm is demonstrated by the consistency of the optimised solutions over 10 runs.

- The Hough transform accurately allows the formation of manufacturable CFS cross-sections, and the algorithm always converges to optimised solutions. The convergence rate for manufacturable cross-sections is slower than for non-manufacturable ones.

- However, the computation time per generation to shape-optimise short to long manufacturable columns is approximately $26 \%$ to $66 \%$ faster than for non-manufacturable ones, because in CUFSM it takes more time to analyse non-manufacturable cross-sections composed of a large number of short elements than to analyse manufacturable ones with a small number of flat segments.

- Introducing manufacturing constraints into shape optimisation algorithms was found to marginally affect the performance of the resulting sections, with the average ultimate compressive stress of the manufacturable columns being within $1.1 \%$ of that of the nonmanufacturable ones.

- The manufacturable cross-sectional shapes were usually found to be similar. "Bean" and closed "Cee" cross-sectional shapes without local stiffeners were mainly found to be the fittest, and likely represent optimum manufacturable or non-manufacturable cross-sectional shapes. Typically, local buckling is prevented by shaping these rounded optimum crosssections rather than forming local stiffeners in the algorithm.

- The optimised singly-symmetric manufacturable cross-sections have a capacity $30 \%$ to $151 \%$ higher than that of the conventional lipped channel sections. 


\section{REFERENCES}

[1] Hancock GJ, Design of cold-formed steel structures (to AS/NZ 4600:2007) - 4th Edition, (Australian Steel Institute), North Sydney, Australia, 2007.

[2] Schafer BW "Review: the direct strength method of cold-formed steel member design", Journal of Constructional Steel Research, 64, 766-778, 2008.

[3] Gilbert BP, Teh LH, Guan H "Self-shape optimisation principles: Optimisation of section capacity for thin-walled profiles", Thin-Walled Structures, 60, 194-204, 2012.

[4] Gilbert BP, Savoyat TJM, Teh LH "Self-shape optimisation application: Optimisation of coldformed steel columns", Thin-Walled Structures, 60, 173-184, 2012.

[5] Liu H, Igusa T, Schafer B "Knowledge-based global optimization of cold-formed steel columns", Thin-Walled Structures, 42, 785-801, 2004.

[6] Leng J, Guest JK, Schafer BW "Shape optimization of cold-formed steel columns", ThinWalled Structures, 49, 1492-1503, 2011.

[7] Moharrami M, Louhghalam A, Tootkaboni M "Optimal folding of cold formed steel cross sections under compression", Thin-Walled Structures, 76, 145-156, 2014.

[8] Leng J, Li Z, Guest JK, Schafer BW, "Constrained shape optimization of cold-formed steel columns", Proceedings of the 21st International Specialty Conference on Cold-Formed Steel Structures, St. Louis, MO; United States, 59-73, 2012.

[9] Leng J, Li Z, Guest JK, Schafer BW, "Shape optimization of cold-formed steel columns with manufacturing constraints and limited number of rollers", Proceedings of the Structural Stability Research Council Annual Stability Conference, 313-331, 2013.

[10] Leng J, Li Z, Guest JK, Schafer BW "Shape optimization of cold-formed steel columns with fabrication and geometric end-use constraints", Thin-Walled Structures, 85, 271-290, 2014.

[11] Franco JMS, Duarte JP, Batista EdM, Landesmann A "Shape Grammar of steel cold-formed sections based on manufacturing rules", Thin-Walled Structures, 79, 218-232, 2014. 
[12] Holland JH, Adaptation in natural and artificial systems, (University of Michigan Press), 1975.

[13] Adeli H, Sarma KC, Evolutionary Computing and the Genetic Algorithm, in: Cost Optimization of Structures, John Wiley \& Sons, Ltd, 2006, pp. 37-52.

[14] Adeli H, Cheng N "Augmented Lagrangian Genetic Algorithm for Structural Optimization", Journal of Aerospace Engineering, 7, 104-118, 1994.

[15] Smith S, Using Evolutionary Algorithms Incorporating the Augmented Lagrangian Penalty Function to Solve Discrete and Continuous Constrained Non-linear Optimal Control Problems, in: P. Collet, C. Fonlupt, J.-K. Hao, E. Lutton, M. Schoenauer (Eds.) Artificial Evolution, Springer Berlin Heidelberg, 2002, pp. 295-308.

[16] Lee K, Application of the Hough transform, University of Massachusetts, Lowell, USA, 2006.

[17] Ragnedda F, Serra M "On optimum thin-walled closed cross section", Structural and Multidisciplinary Optimization, 30, 233-235, 2005.

[18] Belegundu AD, Arorat JS "A computational study of transformation methods for optimal design", AIAA journal, 22, 535-542, 1984.

[19] AS/NZS 4600, Cold-formed steel structures, Standards Australia, Sydney, 2005.

[20] Cheung YK, Finite strip method in structural analysis, (Elsevier), 2013.

[21] Ádány S, Joó AL, Schafer BW "Buckling mode identification of thin-walled members by using cFSM base functions", Thin-Walled Structures, 48, 806-817, 2010.

[22] Schafer B, Ádány S, "Buckling analysis of cold-formed steel members using CUFSM: conventional and constrained finite strip methods", Proceedings of the Eighteenth international specialty conference on cold-formed steel structures (Eds.: R.A. LaBoube, W.W. Yu), St. Louis, Missouri, USA, 39-54, 2006.

[23] Madeira JFA, Dias J, Silvestre N "Multiobjective optimization of cold-formed steel columns", Thin-Walled Structures, 96, 29-38, 2015.

[24] The Lysaght Referee - 32nd Edition, BlueScope Steel Limited, NSW, Australia, 2009. 


\section{FIGURES}

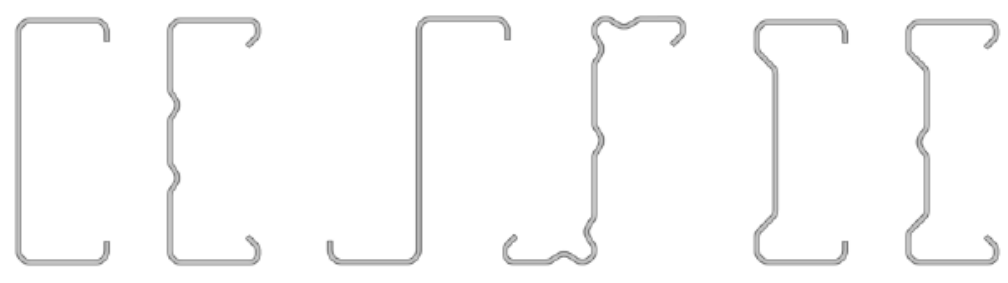

(a)

(b)

(c)

Fig. 1: Conventional CFS profiles with or without stiffeners, (a) Cee, (b) Z and (c) $\Sigma$-sections

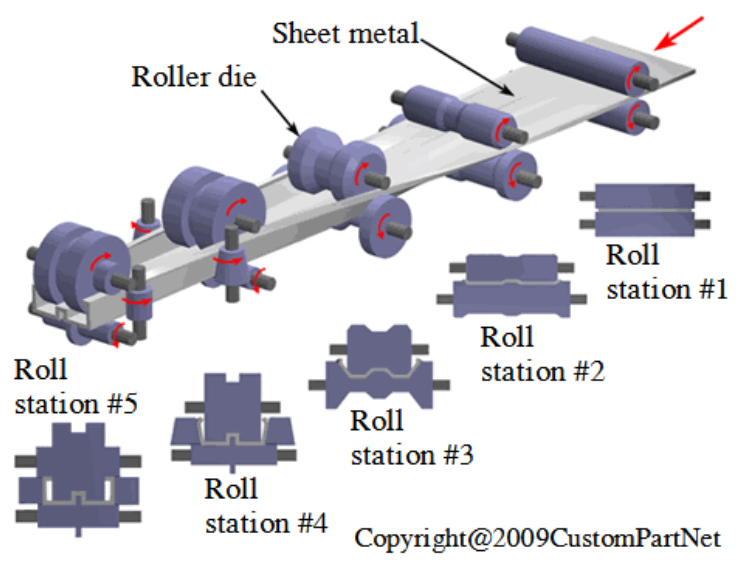

(a) roll-forming

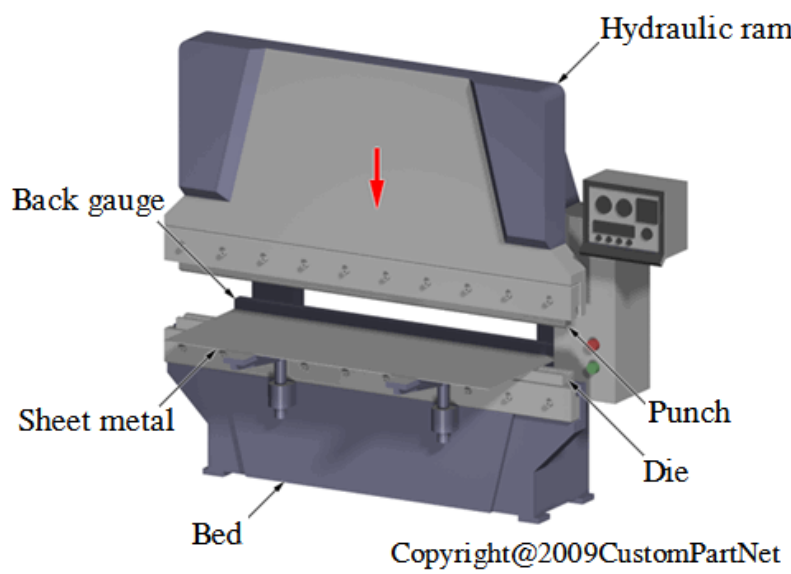

(b) brake-pressing

Fig. 2: Cold-forming processes (Courtesy of CustomPartNet Inc.)

Flat segment length $\geq 10 \mathrm{~mm}$

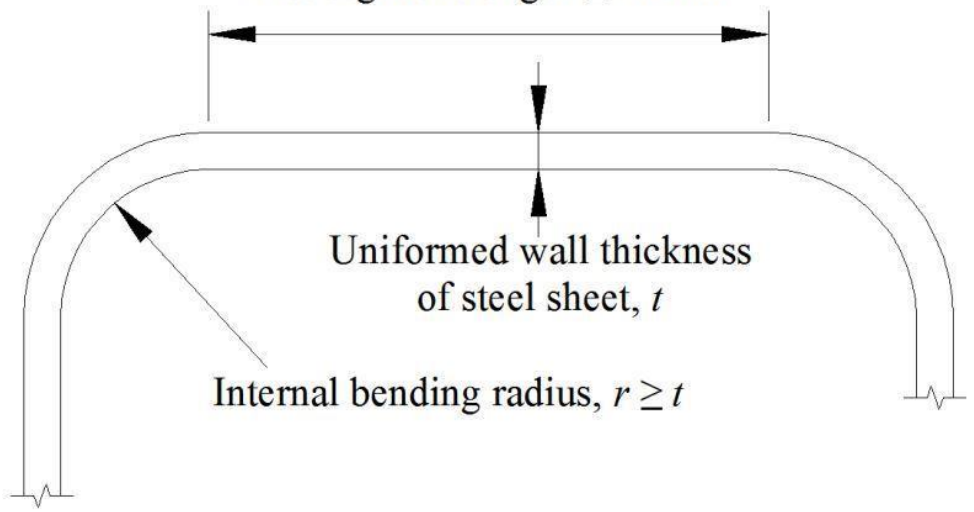

Fig. 3: Manufacturing rules 


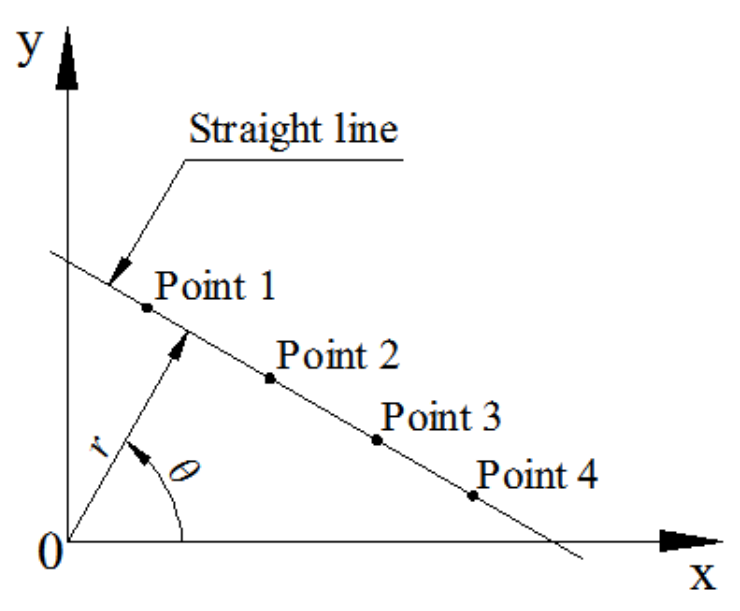

(a) Cartesian coordinate system

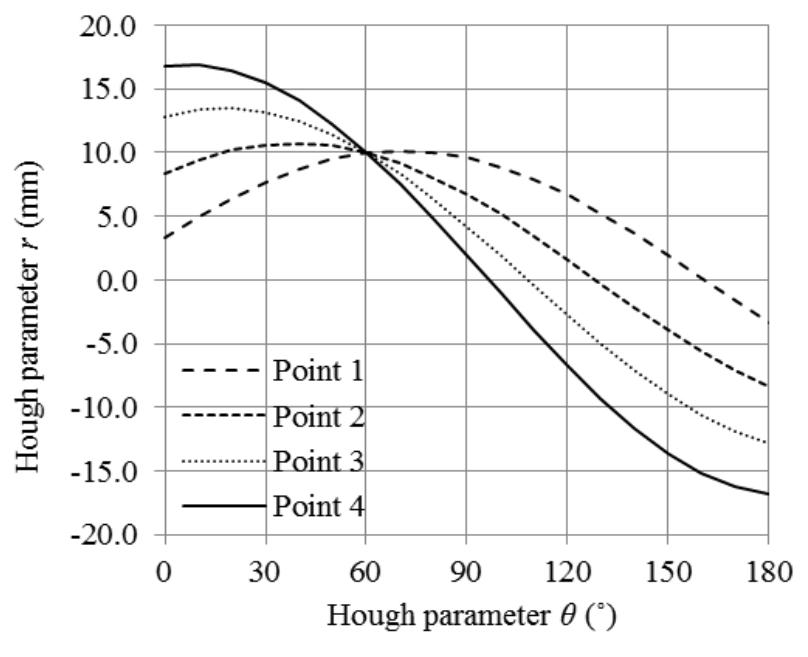

(b) Hough parameter space

Fig. 4. Hough transform from Cartesian space to Hough parametric space

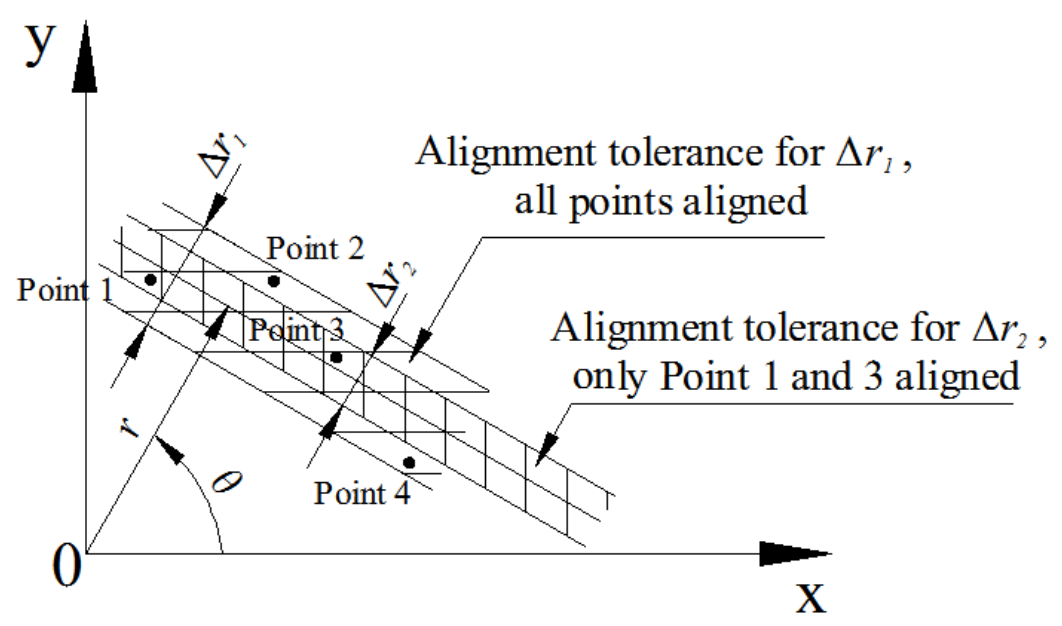

Fig. 5. Alignment tolerance for Hough transform 


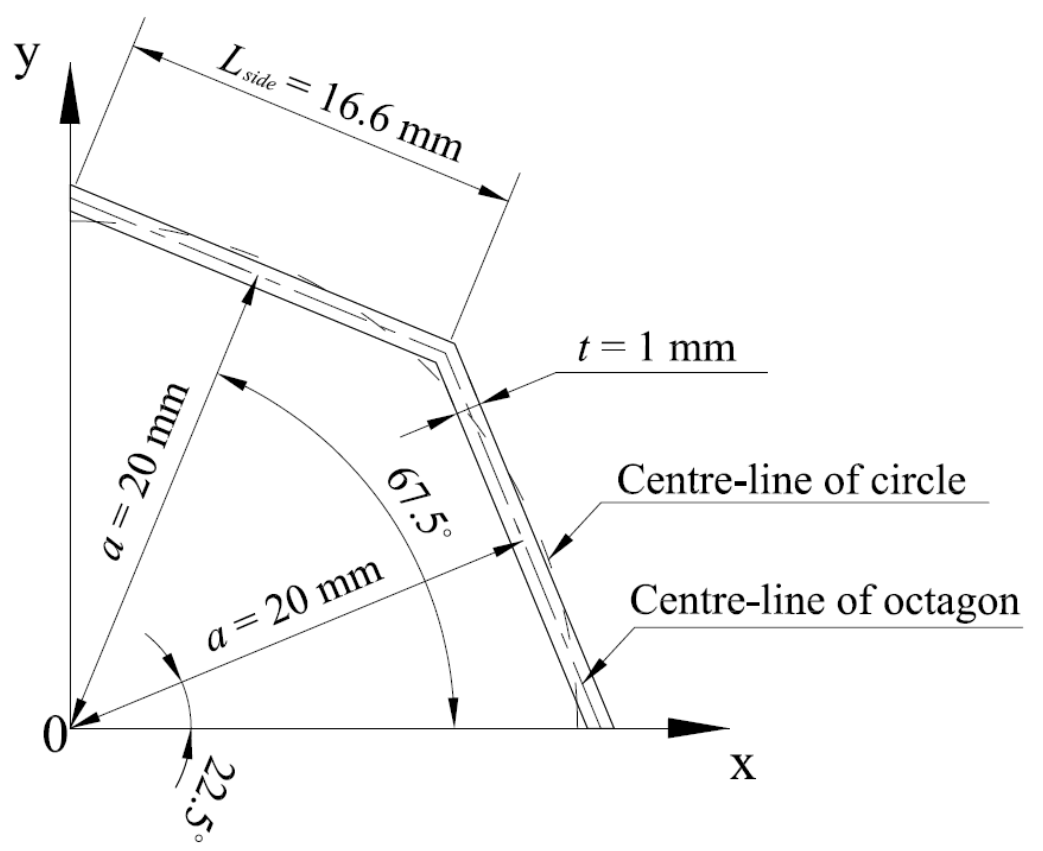

Fig. 6. Optimum octagon, only a quarter section shown

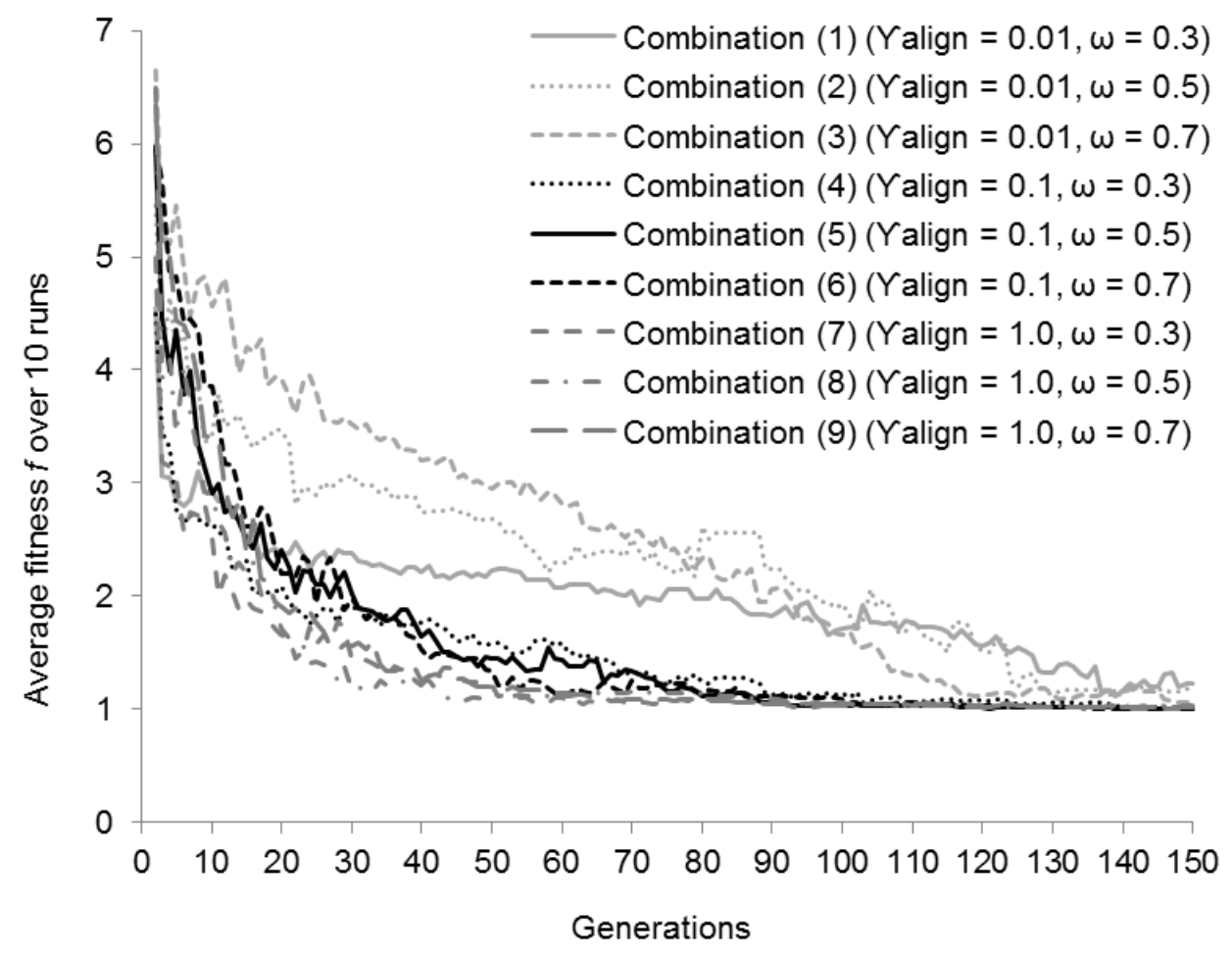

Fig. 7. Average fitness $f$ for parametric study of AL penalty function coefficient $V_{\text {align }}$ and weight $\omega$ 


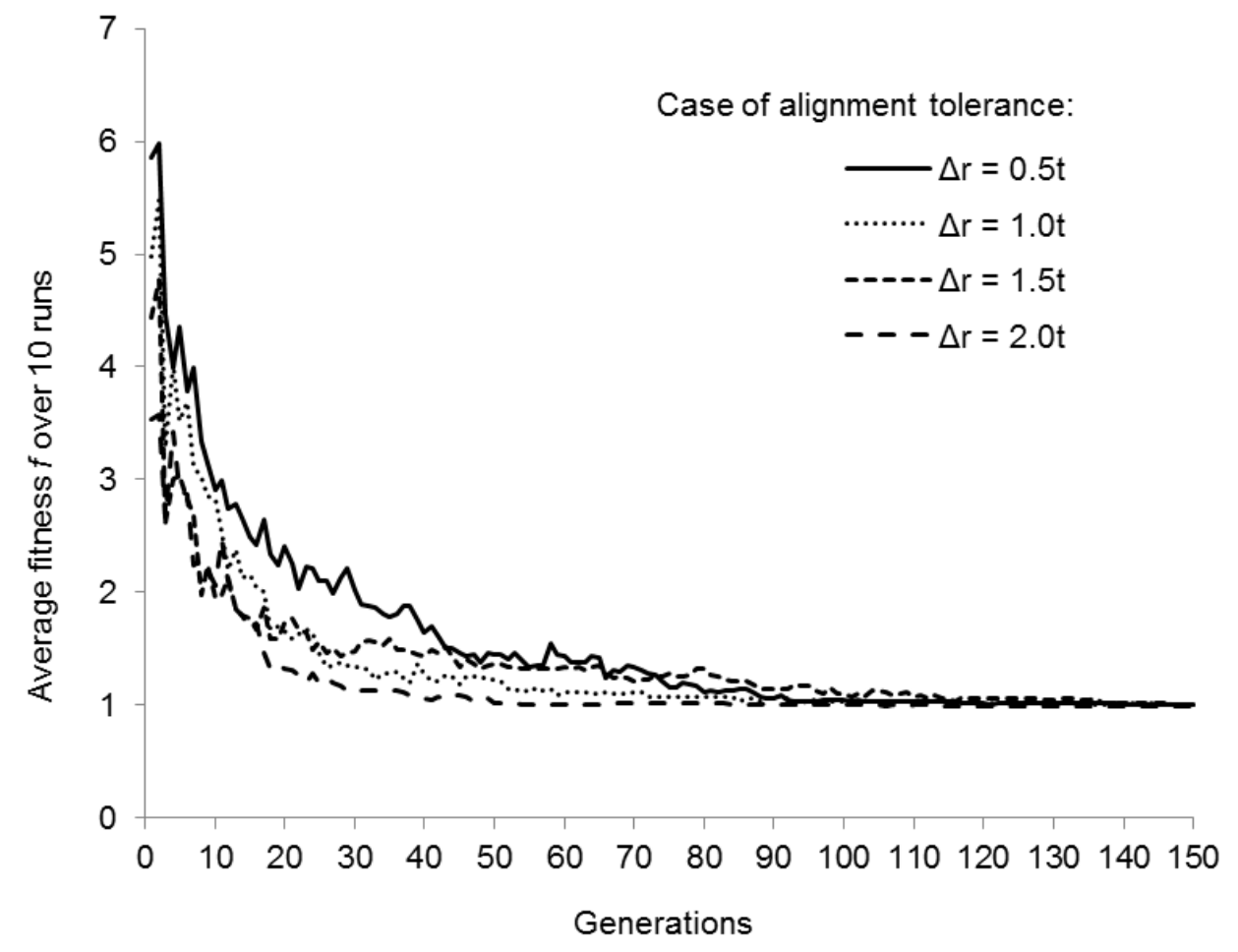

Fig. 8. Average fitness $f$ for parametric study of alignment tolerance $\Delta r$
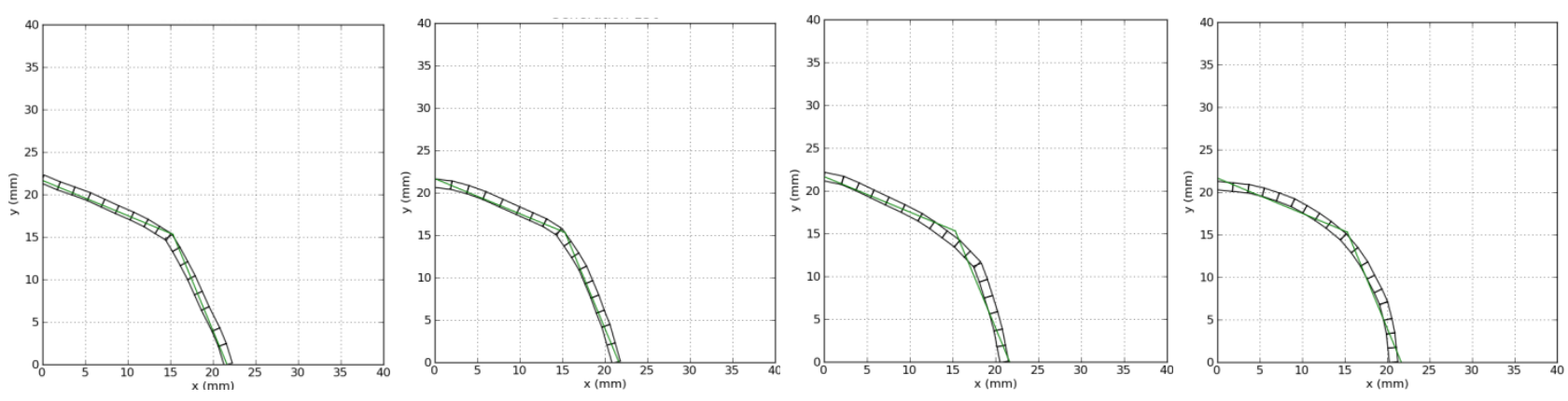
(a) $\Delta r=0.5 t$
$A_{s}=131.7 \mathrm{~mm}^{2}$
$I_{x}=28096.6 \mathrm{~mm}^{4}$
$I_{y}=28076.7 \mathrm{~mm}^{4}$

(b) $\Delta r=1.0 t$

$A_{s}=131.0 \mathrm{~mm}^{2}$

$I_{x}=27989.9 \mathrm{~mm}^{4}$

$I_{y}=27984.8 \mathrm{~mm}^{4}$
(c) $\Delta r=1.5 t$
$A_{s}=130.9 \mathrm{~mm}^{2}$
$I_{x}=28011.2 \mathrm{~mm}^{4}$
$I_{y}=28019.4 \mathrm{~mm}^{4}$
(d) $\Delta r=2.0 t$
$A_{s}=130.4 \mathrm{~mm}^{2}$
$I_{x}=28036.7 \mathrm{~mm}^{4}$
$I_{y}=27999.2 \mathrm{~mm}^{4}$

Fig. 9. Fittest optimised cross-sections at the last generation $\left(150^{\text {th }}\right)$ for all cases of $\Delta r$ from (a) to (d) 


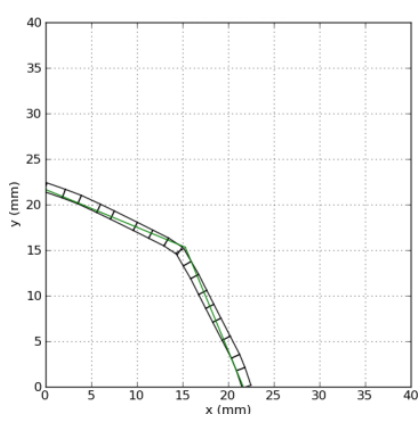

(a) Second fittest

$A_{s}=131.8 \mathrm{~mm}^{2}$

$I_{x}=28024.9 \mathrm{~mm}^{4}$

$I_{y}=28114.3 \mathrm{~mm}^{4}$

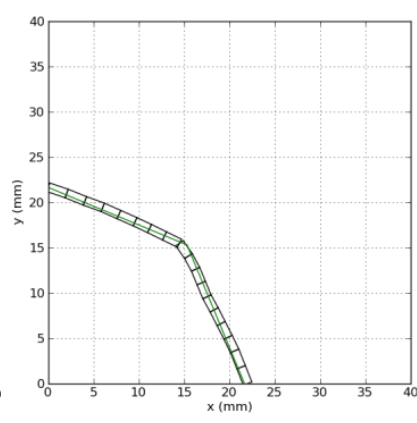

(b) Third least fit $A_{s}=132.1 \mathrm{~mm}^{2}$ $I_{x}=28021.5 \mathrm{~mm}^{4}$ $l_{y}=28246.1 \mathrm{~mm}^{4}$

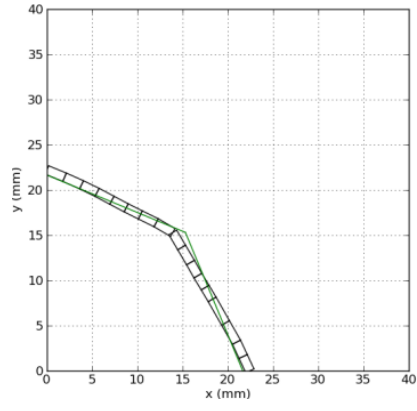

(c) Second least fit $A_{s}=132.3 \mathrm{~mm}^{2}$ $I_{x}=27985.3 \mathrm{~mm}^{4}$ $l_{y}=27972.3 \mathrm{~mm}^{4}$

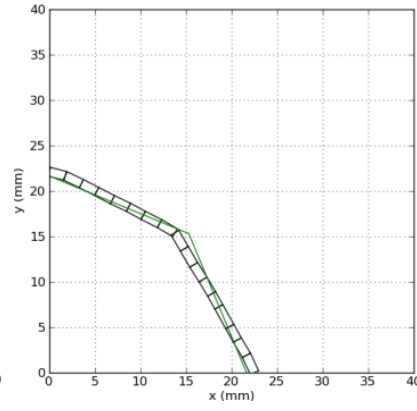

(d) Least fit $A_{s}=132.4 \mathrm{~mm}^{2}$

$I_{x}=28074.3 \mathrm{~mm}^{4}$

$l_{y}=28034.9 \mathrm{~mm}^{4}$

Fig. 10. Optimised cross-sections at the last generation $\left(150^{\text {th }}\right)$, for the second fittest $(\mathrm{a})$, third fittest (b), second least fit (c) and least fit (d) cross-sections out of 10 runs for the case $\Delta r=0.5 t$

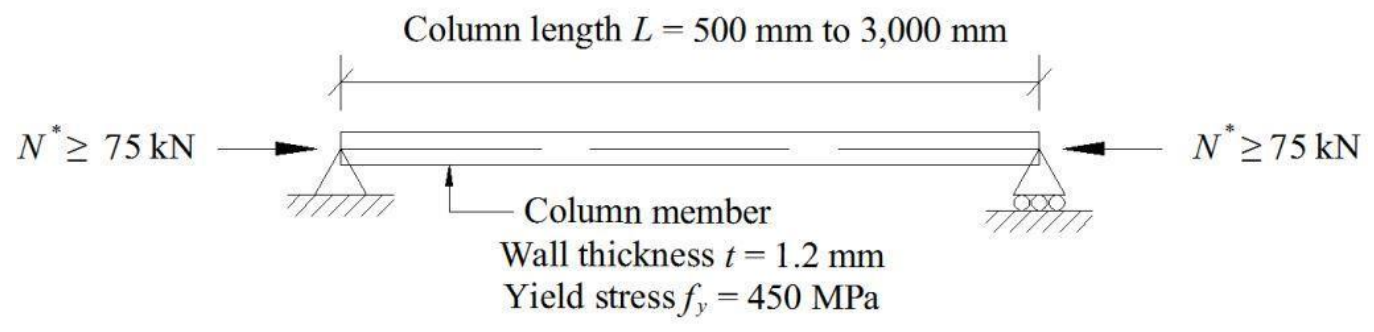

Fig. 11: Optimisation problem

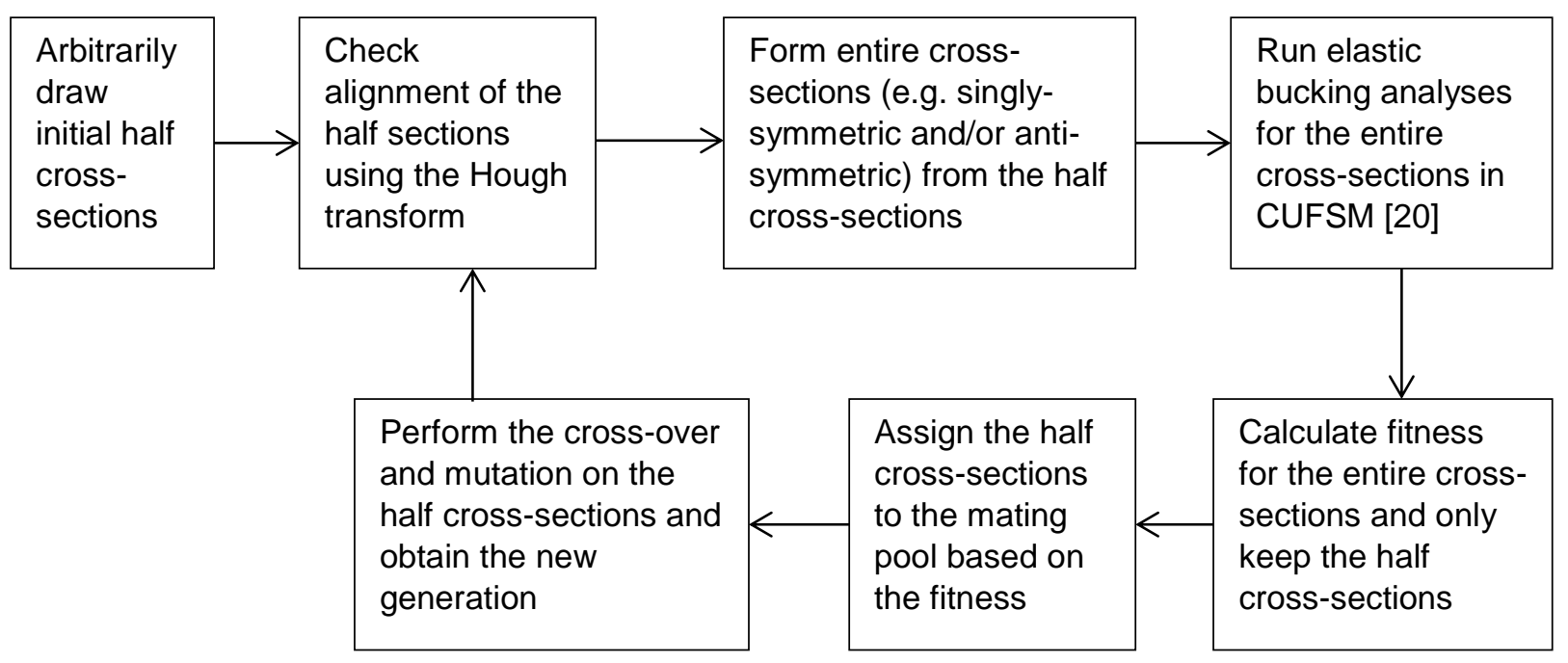

Fig. 12: Simplified flowchart of the algorithm 


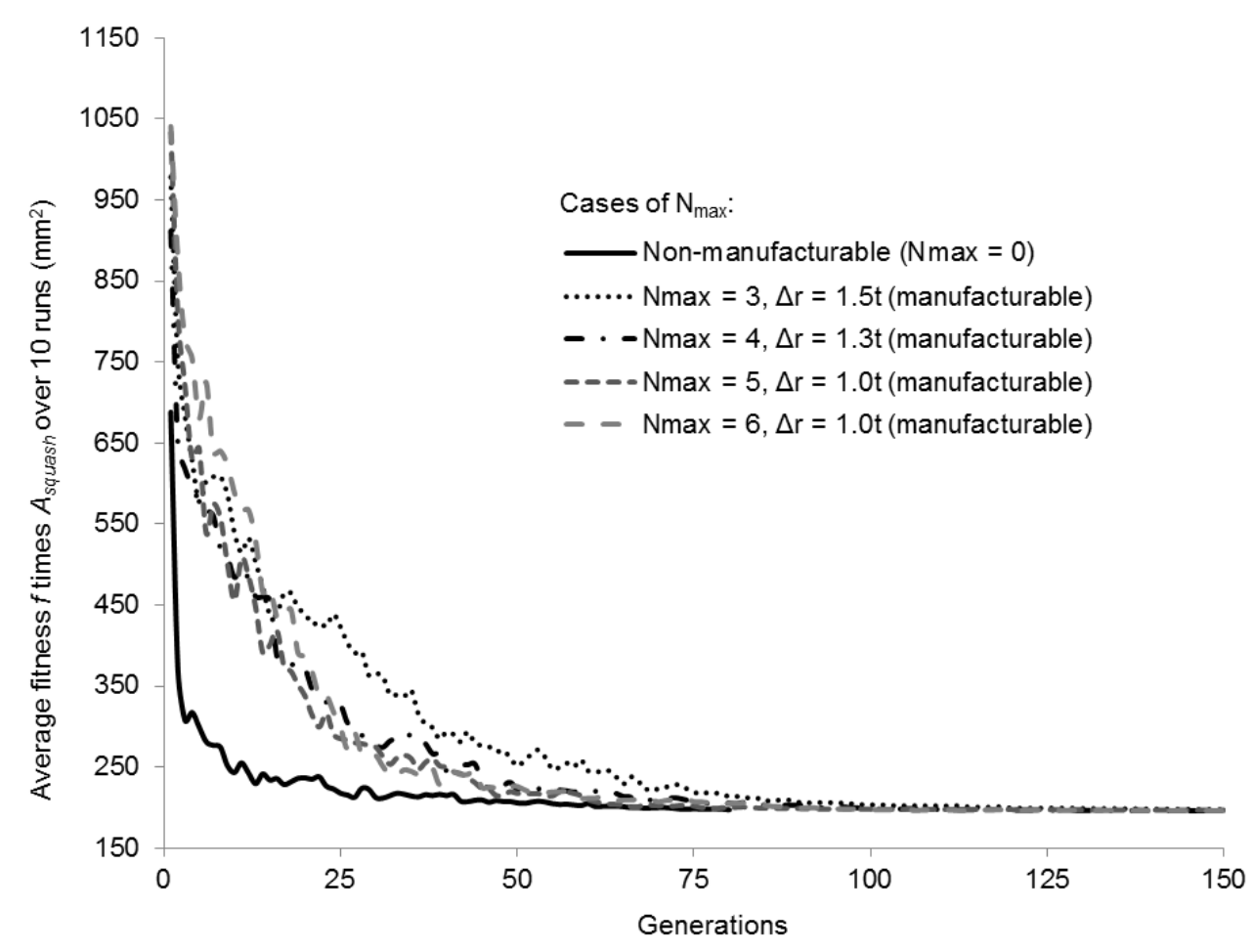

(a)

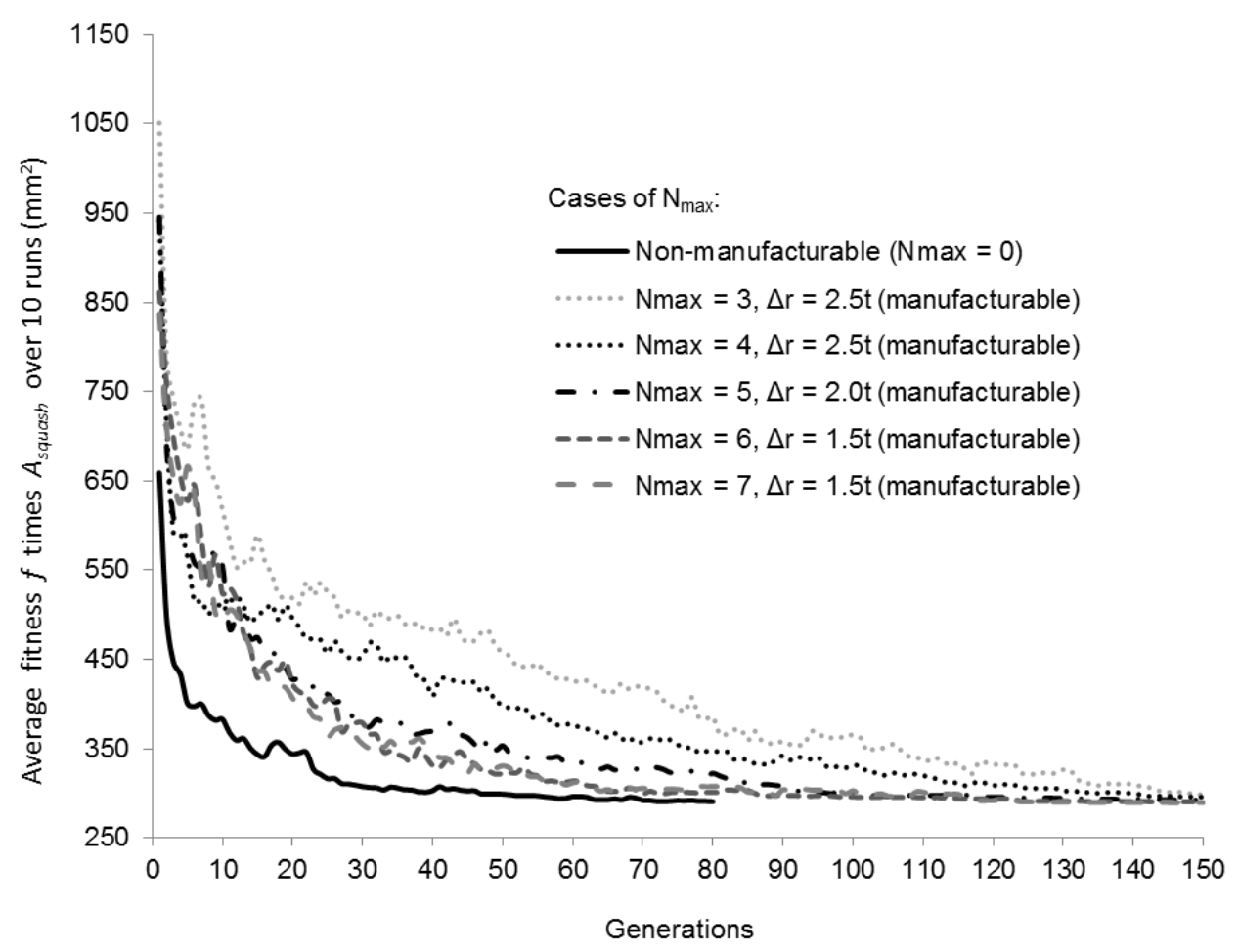

(b) 


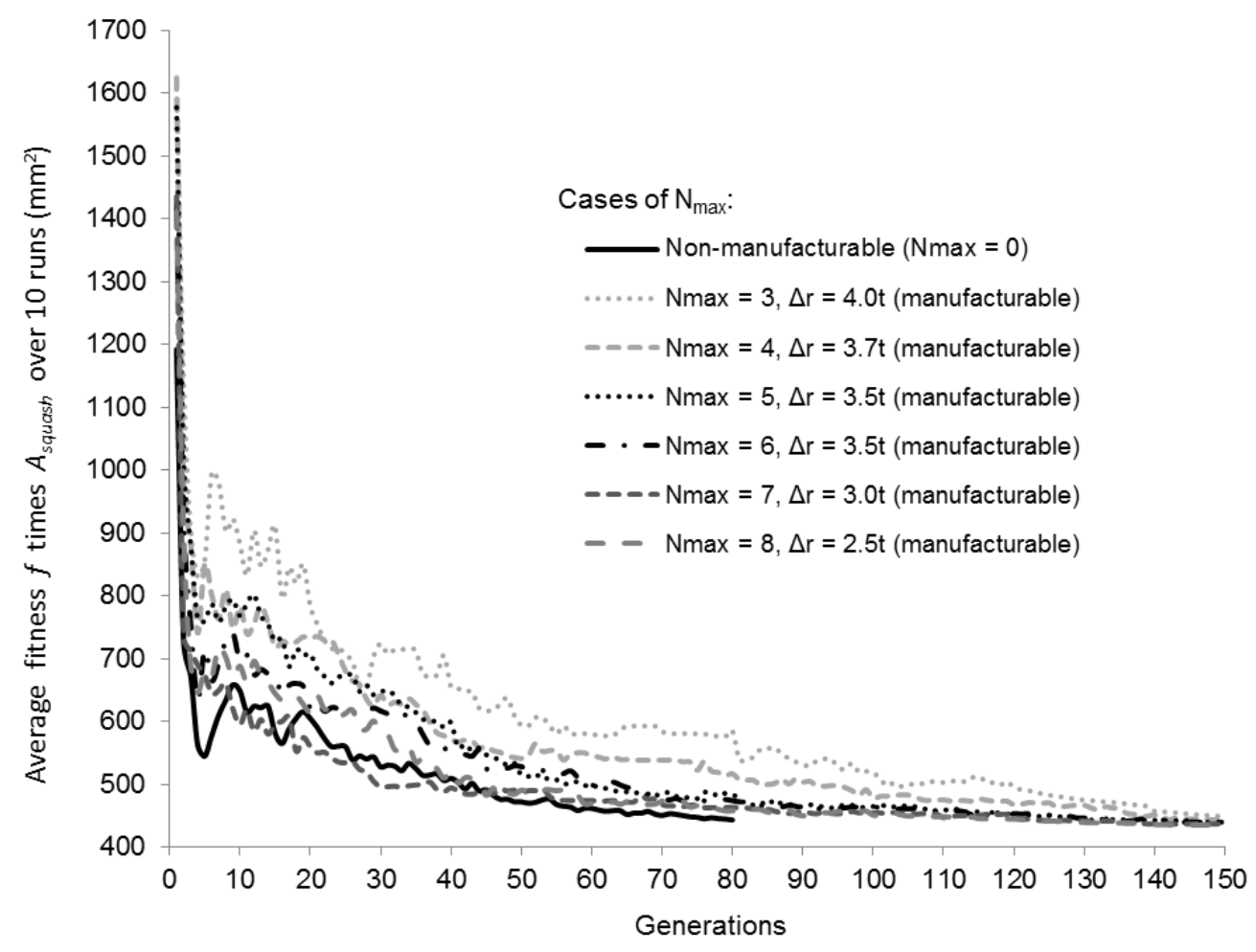

(c)

Fig. 13: Average fitness $f$ for the (a) $500 \mathrm{~mm}$, (b) $1,500 \mathrm{~mm}$ and (c) $3,000 \mathrm{~mm}$ long columns
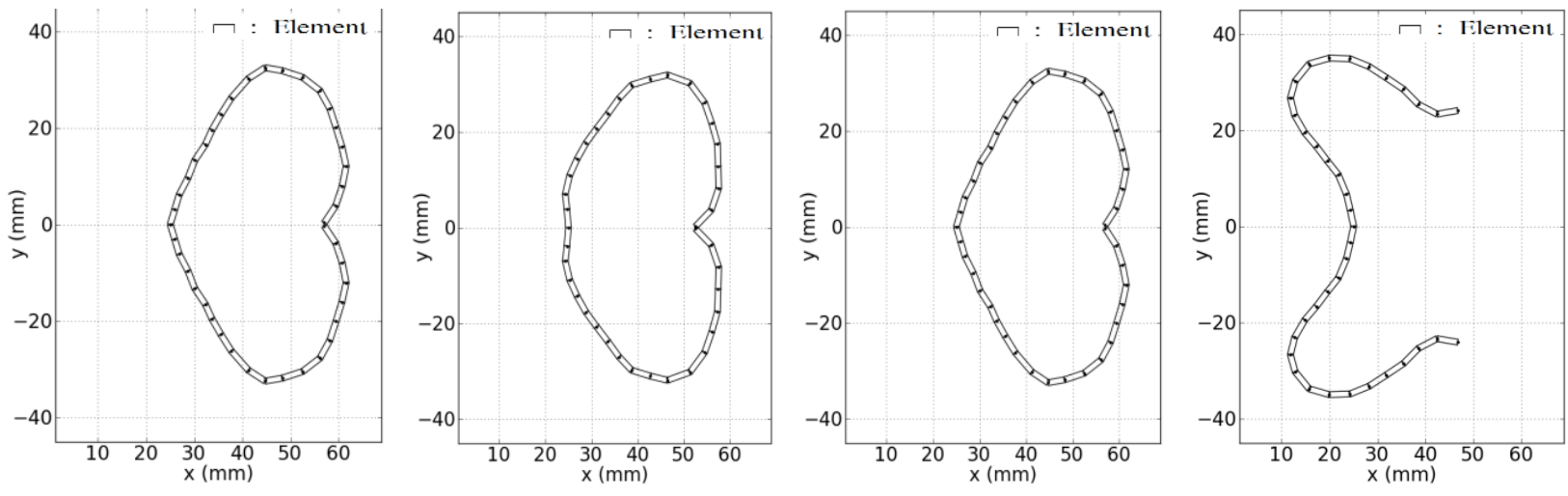
(a) $A_{s}=193.8 \mathrm{~mm}^{2}$
$N_{c}=75.16 \mathrm{kN}$
$N_{c} / A_{s}=387.8 \mathrm{MPa}$

(b) $A_{s}=193.7 \mathrm{~mm}^{2}$

$$
\begin{aligned}
& N_{c}=75.07 \mathrm{kN} \\
& N_{d} / A_{s}=387.6 \mathrm{MPa}
\end{aligned}
$$

(c) $A_{s}=195.8 \mathrm{~mm}^{2}$

$N_{c}=74.90 \mathrm{kN}$

$N_{d} / A_{s}=382.5 \mathrm{MPa}$ (d) $A_{s}=196.9 \mathrm{~mm}^{2}$

$N_{c}=74.81 \mathrm{kN}$

$N_{d} / A_{s}=379.9 \mathrm{MPa}$

Fig. 14: Optimised cross-sections for the $500 \mathrm{~mm}$ long columns and the non-manufacturable case,

(a, b) fittest cross-sections and (c, d) least fit cross-sections 

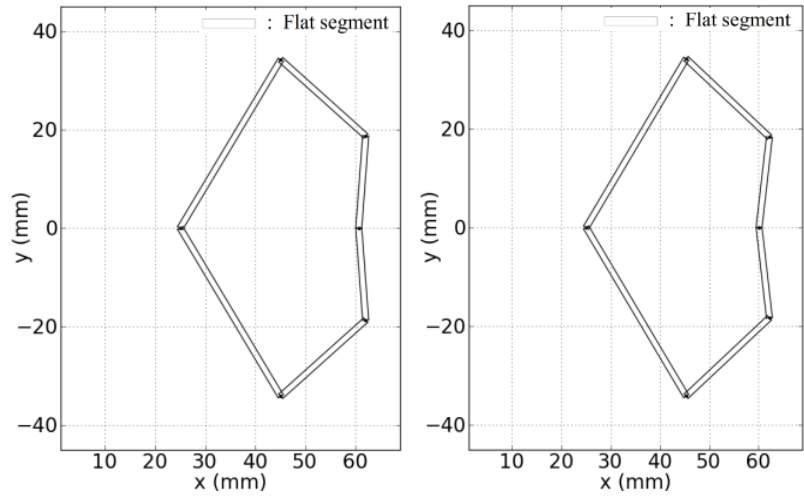
(a) $A_{s}=195.1 \mathrm{~mm}^{2}$
$N_{c}=74.98 \mathrm{kN}$
$N_{c} / A_{s}=384.3 \mathrm{MPa}$

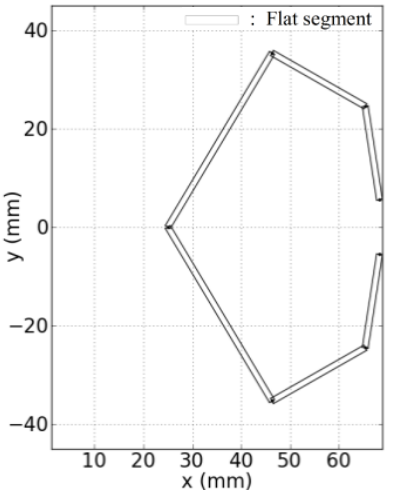

(c) $A_{s}=197.7 \mathrm{~mm}^{2}$ $N_{c}=74.97 \mathrm{kN}$

$N_{d} A_{s}=379.2 \mathrm{MPa}$

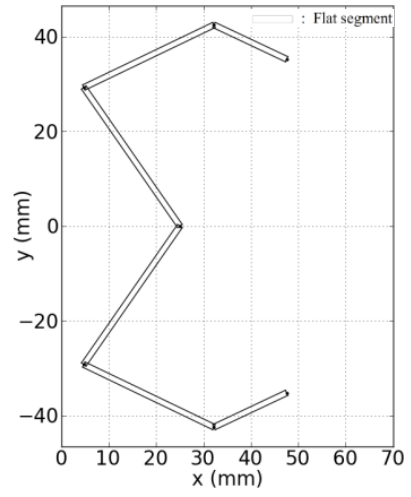

(d) $A_{s}=199.4 \mathrm{~mm}^{2}$ $N_{c}=75.00 \mathrm{kN}$ $N_{d} / A_{s}=376.1 \mathrm{MPa}$

Fig. 15: Optimised cross-sections for the $500 \mathrm{~mm}$ long columns and $N_{\max }=3,(\mathrm{a}, \mathrm{b})$ fittest crosssections and (c, d) least fit cross-sections

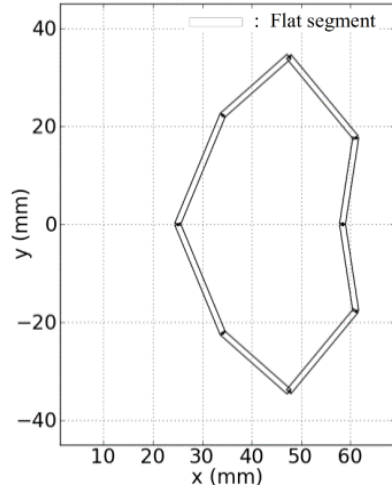

(a) $A_{s}=194.7 \mathrm{~mm}^{2}$ $N_{c}=75.00 \mathrm{kN}$

$N_{d} / A_{s}=385.2 \mathrm{MPa}$

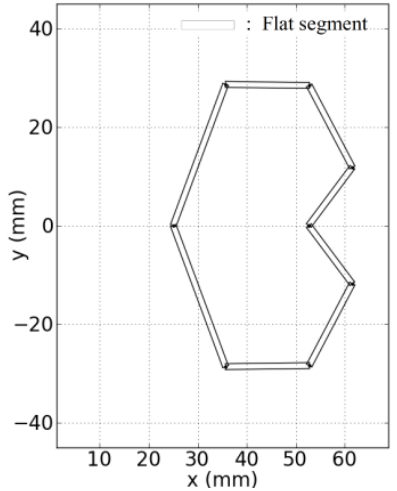

(b) $A_{s}=194.8 \mathrm{~mm}^{2}$ $N_{c}=75.00 \mathrm{kN}$ $N_{d} A_{s}=384.9 \mathrm{MPa}$

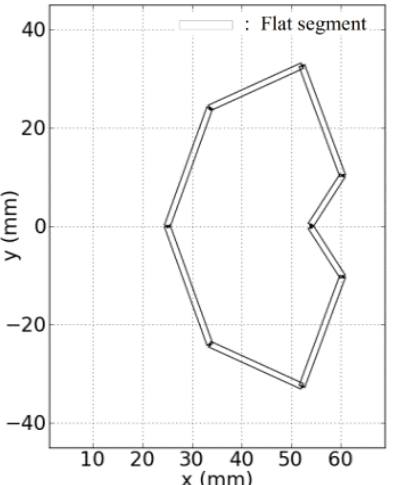

(c) $A_{s}=196.0 \mathrm{~mm}^{2}$ $N_{c}=75.04 \mathrm{kN}$ $N_{d} / A_{s}=383.0 \mathrm{MPa}$

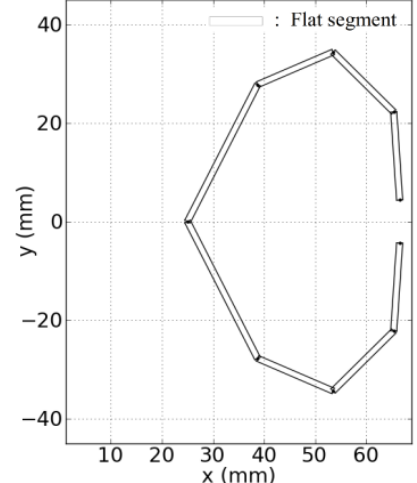

(d) $A_{s}=196.9 \mathrm{~mm}^{2}$ $N_{c}=75.01 \mathrm{kN}$ $N_{d} A_{s}=380.9 \mathrm{MPa}$

Fig. 16: Optimised cross-sections for the $500 \mathrm{~mm}$ long columns and $N_{\max }=4,(\mathrm{a}, \mathrm{b})$ fittest crosssections and (c, d) least fit cross-sections 


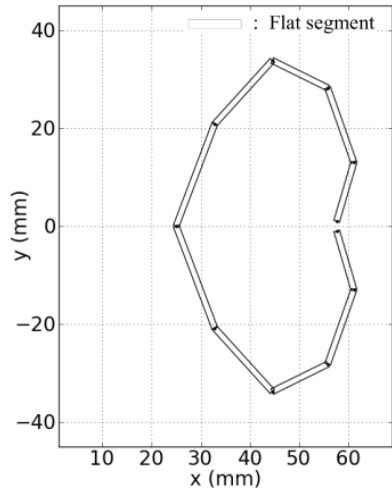

(a) $A_{s}=194.0 \mathrm{~mm}^{2}$ $N_{c}=74.95 \mathrm{kN}$

$N_{d} A_{s}=386.3 \mathrm{MPa}$

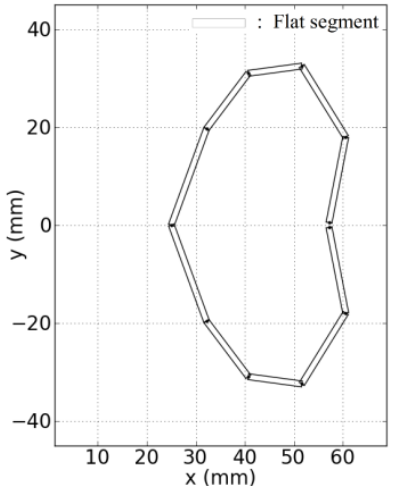

(b) $A_{s}=194.2 \mathrm{~mm}^{2}$ $N_{c}=75.00 \mathrm{kN}$

$N_{d} / A_{s}=386.2 \mathrm{MPa}$

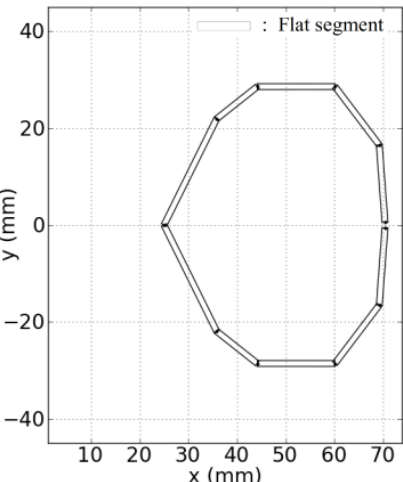

(c) $A_{s}=197.2 \mathrm{~mm}^{2}$ $\mathrm{N}_{C}=75.00 \mathrm{kN}$

$N_{d} A_{s}=380.2 \mathrm{MPa}$

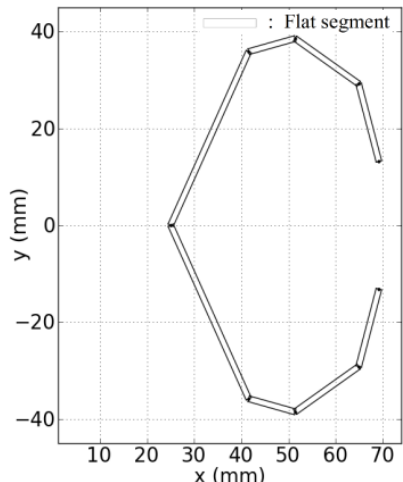

(d) $A_{s}=198.4 \mathrm{~mm}^{2}$ $N_{c}=75.05 \mathrm{kN}$

$N_{d} A_{s}=378.3 \mathrm{MPa}$

Fig. 17: Optimised cross-sections for the $500 \mathrm{~mm}$ long columns and $N_{\max }=5,(\mathrm{a}, \mathrm{b})$ fittest crosssections and (c, d) least fit cross-sections

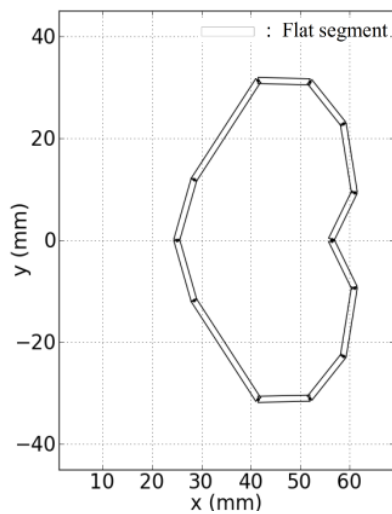

(a) $A_{s}=193.9 \mathrm{~mm}^{2}$ $N_{c}=74.93 \mathrm{kN}$ $N_{d} / A_{s}=386.4 \mathrm{MPa}$

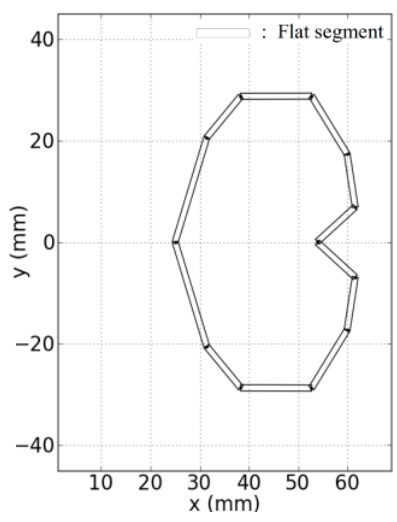

(b) $A_{s}=194.2 \mathrm{~mm}^{2}$ $N_{c}=75.01 \mathrm{kN}$

$N_{d} / A_{s}=386.3 \mathrm{MPa}$

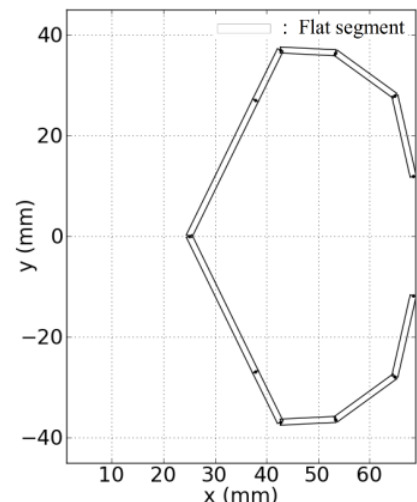

(c) $A_{s}=197.9 \mathrm{~mm}^{2}$ $N_{c}=75.01 \mathrm{kN}$ $N_{d} / A_{s}=379.0 \mathrm{MPa}$

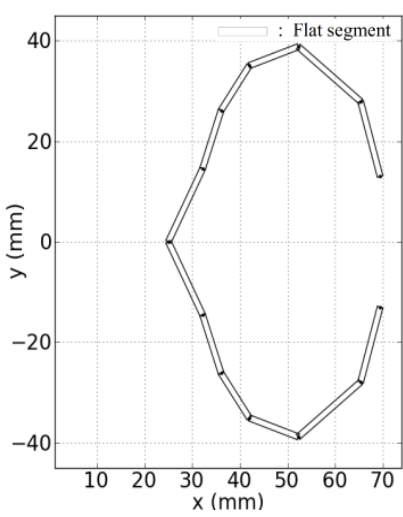

(d) $A_{s}=198.2 \mathrm{~mm}^{2}$

$N_{c}=74.98 \mathrm{kN}$

$N_{d} A_{s}=378.3 \mathrm{MPa}$

Fig. 18: Optimised cross-sections for the $500 \mathrm{~mm}$ long columns and $N_{\max }=6$, $(\mathrm{a}, \mathrm{b})$ fittest crosssections and (c, d) least fit cross-sections 


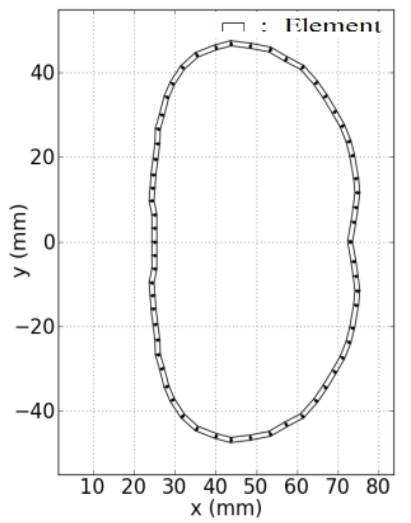

(a) $A_{s}=287.6 \mathrm{~mm}^{2}$ $N_{c}=75.05 \mathrm{kN}$

$N_{c} / A_{s}=261.0 \mathrm{MPa}$

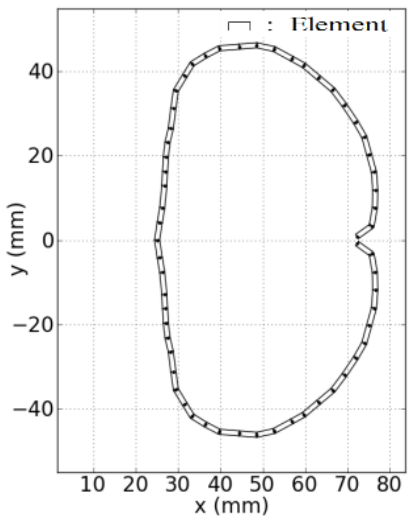

(b) $A_{s}=287.7 \mathrm{~mm}^{2}$ $N_{c}=75.06 \mathrm{kN}$

$N_{c} / A_{s}=261.0 \mathrm{MPa}$

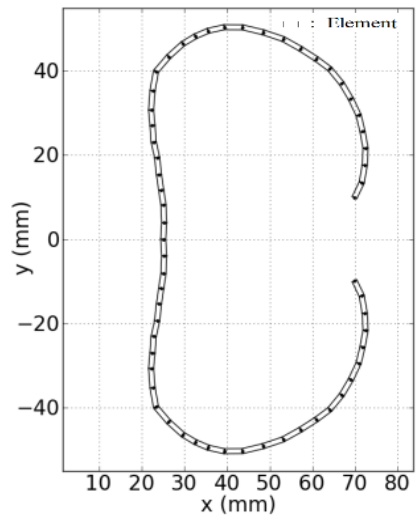

(c) $A_{s}=289.0 \mathrm{~mm}^{2}$ $N_{c}=74.95 \mathrm{kN}$ $N_{d} / A_{s}=259.3 \mathrm{MPa}$

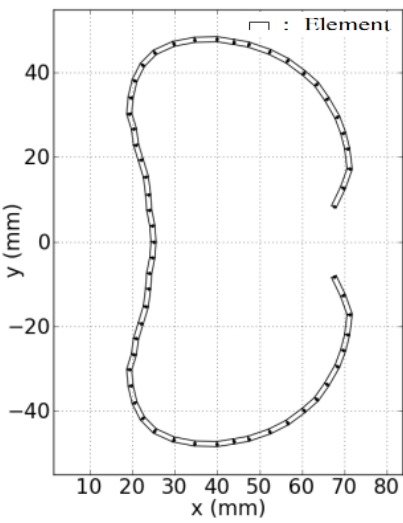

(d) $A_{s}=288.8 \mathrm{~mm}^{2}$ $N_{c}=74.84 \mathrm{kN}$

$N_{c} / A_{s}=259.1 \mathrm{MPa}$

Fig. 19: Optimised cross-sections for the $1,500 \mathrm{~mm}$ long columns and the non-manufacturable case,

$(a, b)$ fittest cross-sections and (c, d) least fit cross-sections

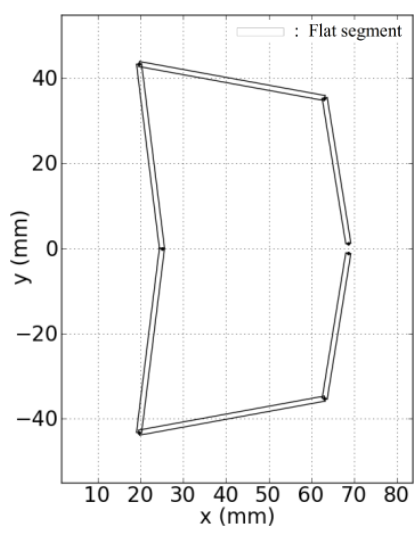

(a) $A_{s}=292.5 \mathrm{~mm}^{2}$ $N_{c}=75.03 \mathrm{kN}$ $N_{c} / A_{s}=256.5 \mathrm{MPa}$

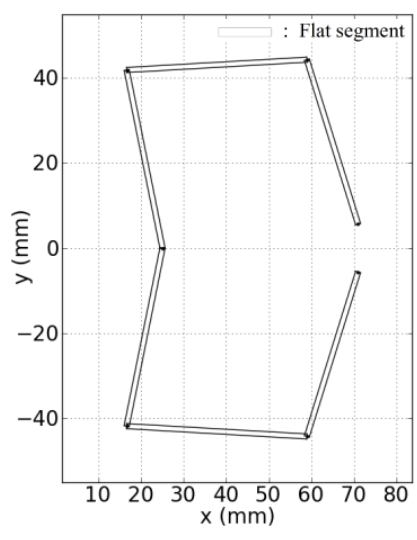

(b) $A_{s}=296.3 \mathrm{~mm}^{2}$ $N_{c}=75.87 \mathrm{kN}$ $N_{c} / A_{s}=256.1 \mathrm{MPa}$
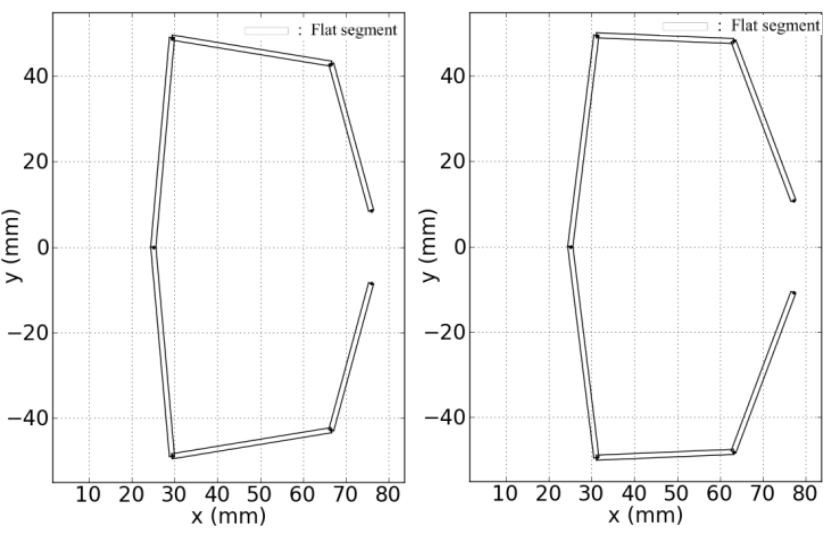

(c) $A_{s}=296.1 \mathrm{~mm}^{2}$ $N_{c}=74.52 \mathrm{kN}$ $N_{c} / A_{s}=251.7 \mathrm{MPa}$ (d) $A_{s}=301.7 \mathrm{~mm}^{2}$ $N_{c}=75.29 \mathrm{kN}$ $N_{c} / A_{s}=249.6 \mathrm{MPa}$

Fig. 20: Optimised cross-sections for the $1,500 \mathrm{~mm}$ long columns and $N_{\max }=3,(\mathrm{a}, \mathrm{b})$ fittest crosssections and (c, d) least fit cross-sections 


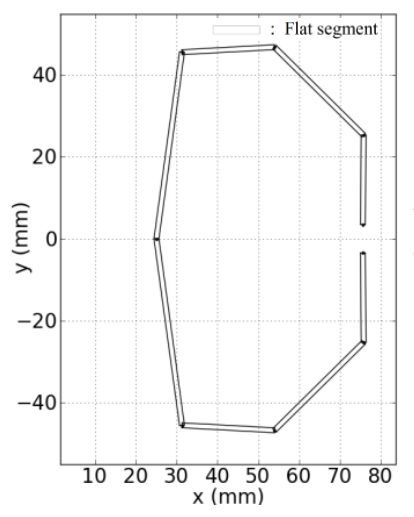

(a) $A_{s}=291.0 \mathrm{~mm}^{2}$ $N_{c}=75.08 \mathrm{kN}$

$N_{C} / A_{s}=258.0 \mathrm{MPa}$

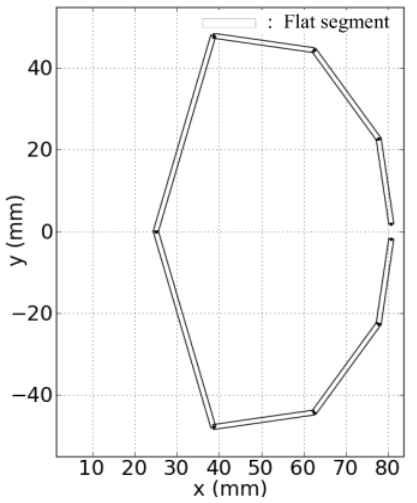

(b) $A_{s}=291.6 \mathrm{~mm}^{2}$ $N_{c}=75.17 \mathrm{kN}$

$N_{d} / A_{s}=257.8 \mathrm{MPa}$

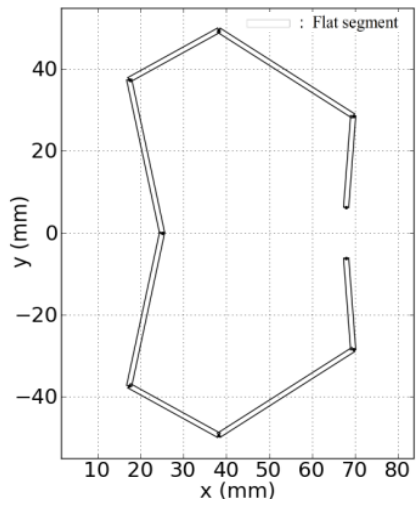

(c) $A_{s}=294.0 \mathrm{~mm}^{2}$ $N_{c}=74.96 \mathrm{kN}$

$N_{d} / A_{s}=255.0 \mathrm{MPa}$

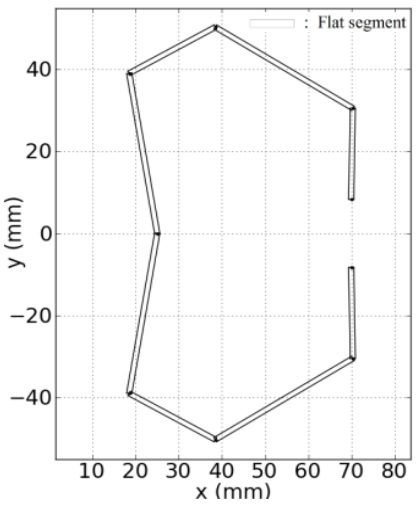

(d) $A_{s}=293.5 \mathrm{~mm}^{2}$ $N_{c}=74.67 \mathrm{kN}$

$N_{c} / A_{s}=254.4 \mathrm{MPa}$

Fig. 21: Optimised cross-sections for the $1,500 \mathrm{~mm}$ long columns and $N_{\max }=4$, (a, b) fittest crosssections and (c, d) least fit cross-sections

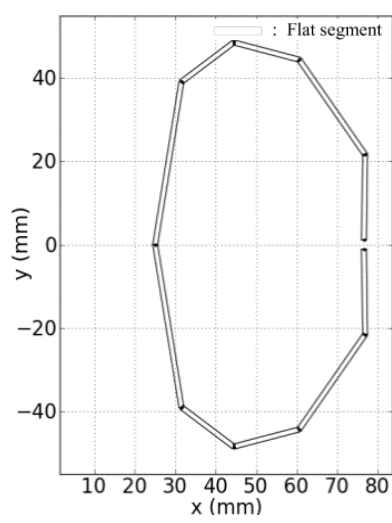

(a) $A_{s}=290.1 \mathrm{~mm}^{2}$ $N_{c}=75.22 \mathrm{kN}$

$N_{d} A_{s}=259.3 \mathrm{MPa}$

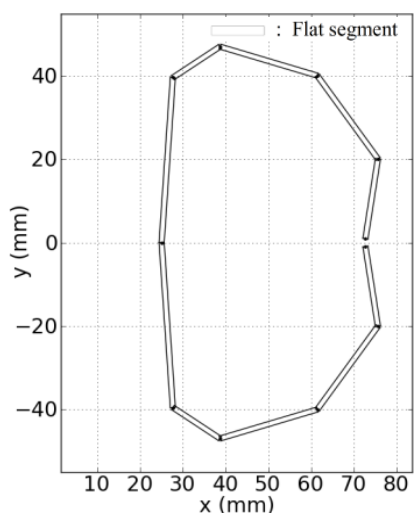

(b) $A_{s}=289.8 \mathrm{~mm}^{2}$ $N_{c}=75.13 \mathrm{kN}$

$N_{d} / A_{s}=259.3 \mathrm{MPa}$

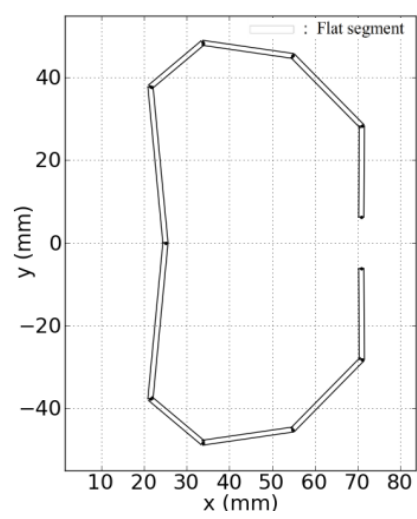

(c) $A_{s}=290.3 \mathrm{~mm}^{2}$ $N_{c}=74.92 \mathrm{kN}$ $N_{d} / A_{s}=258.0 \mathrm{MPa}$

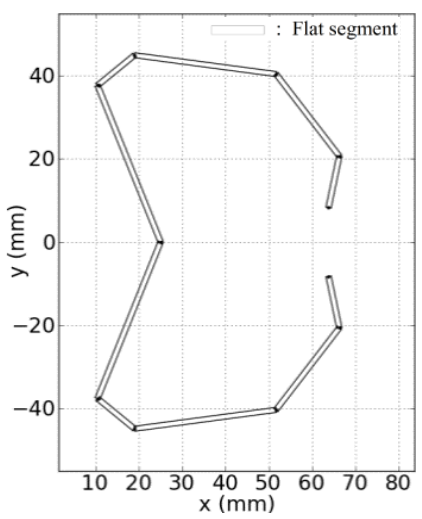

(d) $A_{s}=292.6 \mathrm{~mm}^{2}$ $N_{c}=75.02 \mathrm{kN}$

$N_{d} / A_{s}=256.4 \mathrm{MPa}$

Fig. 22: Optimised cross-sections for the $1,500 \mathrm{~mm}$ long columns and $N_{\max }=5,(\mathrm{a}, \mathrm{b})$ fittest crosssections and (c, d) least fit cross-sections 


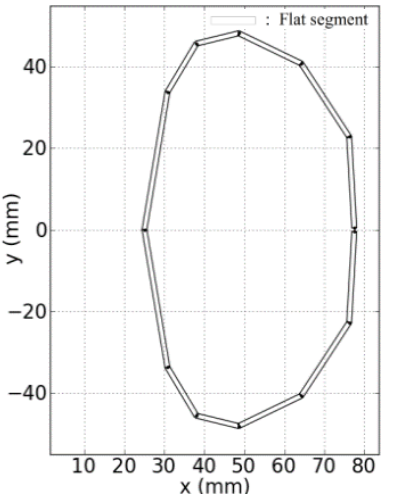

(a) $A_{s}=288.7 \mathrm{~mm}^{2}$

$N_{c}=75.01 \mathrm{kN}$

$N_{c} / A_{s}=259.8 \mathrm{MPa}$

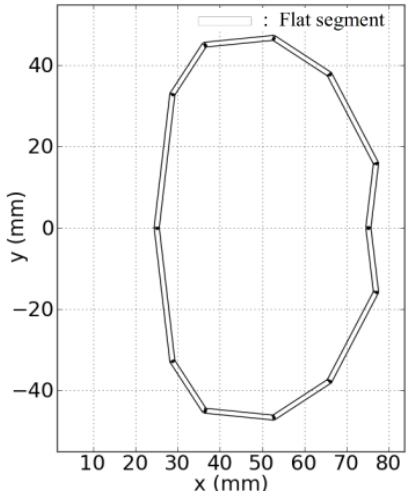

(b) $A_{s}=288.8 \mathrm{~mm}^{2}$ $N_{c}=75.02 \mathrm{kN}$

$N_{c} / A_{s}=259.8 \mathrm{MPa}$

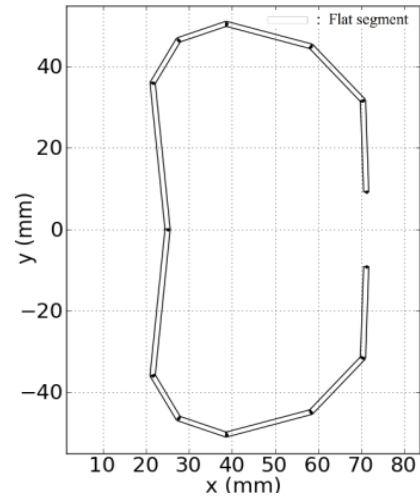

(c) $A_{s}=290.4 \mathrm{~mm}^{2}$ $N_{c}=74.93 \mathrm{kN}$

$N_{d} A_{s}=258.1 \mathrm{MPa}$

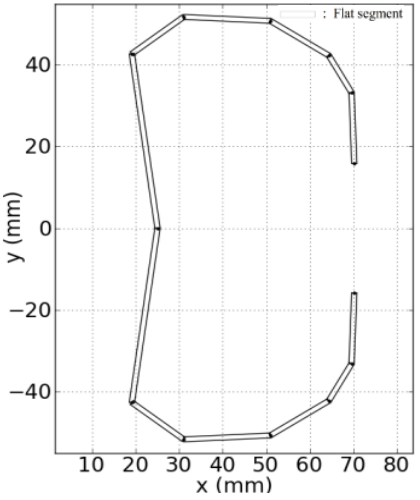

(d) $A_{s}=292.1 \mathrm{~mm}^{2}$ $N_{c}=74.98 \mathrm{kN}$

$N_{d} / A_{s}=256.7 \mathrm{MPa}$

Fig. 23: Optimised cross-sections for the $1,500 \mathrm{~mm}$ long columns and $N_{\max }=6,(\mathrm{a}, \mathrm{b})$ fittest crosssections and (c, d) least fit cross-sections

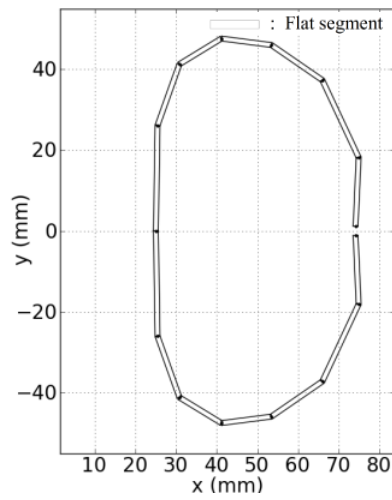

(a) $A_{s}=288.3 \mathrm{~mm}^{2}$ $N_{c}=75.02 \mathrm{kN}$ $N_{\delta} / A_{s}=260.2 \mathrm{MPa}$

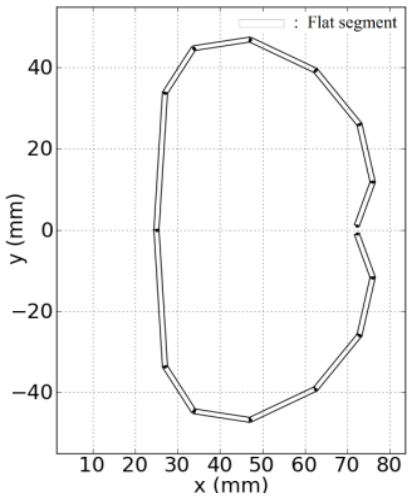

(b) $A_{s}=288.4 \mathrm{~mm}^{2}$ $N_{c}=75.02 \mathrm{kN}$ $N_{c} / A_{s}=260.1 \mathrm{MPa}$

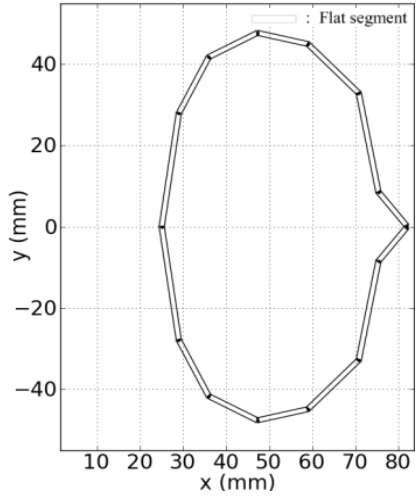

(c) $A_{s}=289.8 \mathrm{~mm}^{2}$ $N_{c}=75.00 \mathrm{kN}$ $N_{d} A_{s}=258.8 \mathrm{MPa}$

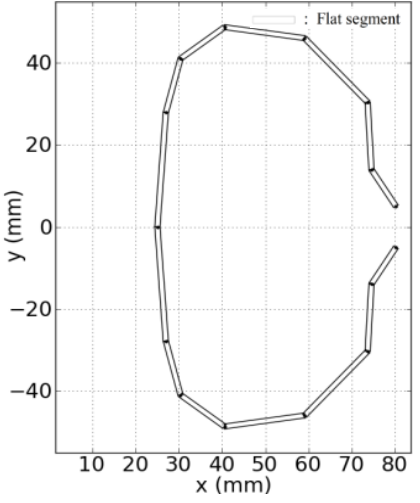

(d) $A_{s}=291.5 \mathrm{~mm}^{2}$ $N_{c}=74.97 \mathrm{kN}$ $N_{d} / A_{s}=257.2 \mathrm{MPa}$

Fig. 24: Optimised cross-sections for the $1,500 \mathrm{~mm}$ long columns and $N_{\max }=7,(\mathrm{a}, \mathrm{b})$ fittest crosssections and (c, d) least fit cross-sections 

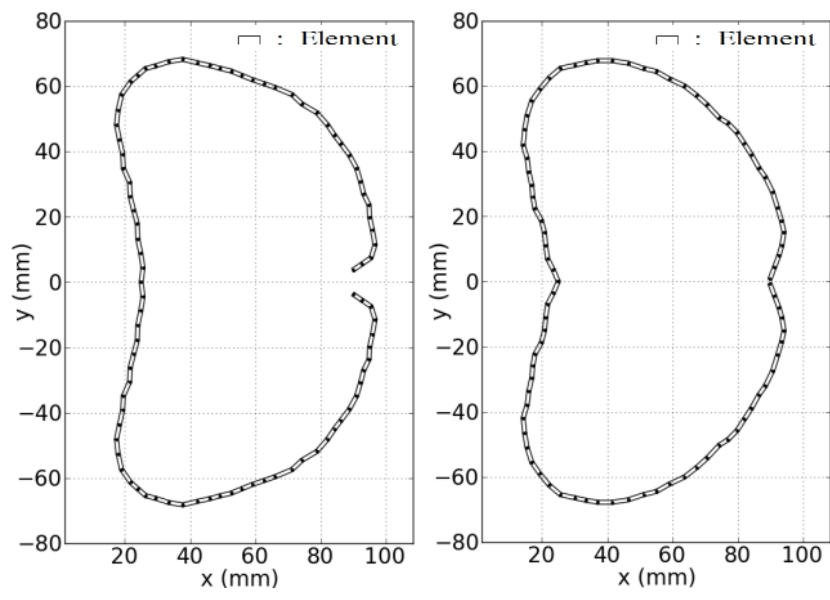

(a) $A_{s}=437.4 \mathrm{~mm}^{2}$
$N_{c}=75.86 \mathrm{kN}$
$N_{d} A_{s}=173.4 \mathrm{MPa}$ (b) $A_{s}=435.8 \mathrm{~mm}^{2}$ $N_{c}=75.52 \mathrm{kN}$

$N_{d} / A_{s}=173.3 \mathrm{MPa}$

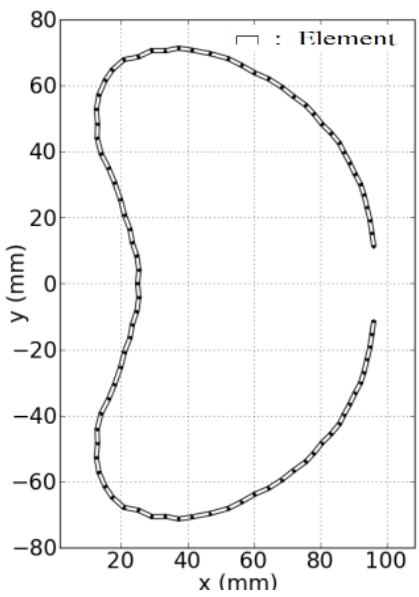

(c) $A_{s}=434.2 \mathrm{~mm}^{2}$ $N_{c}=74.78 \mathrm{kN}$ $N_{d} A_{s}=172.2 \mathrm{MPa}$

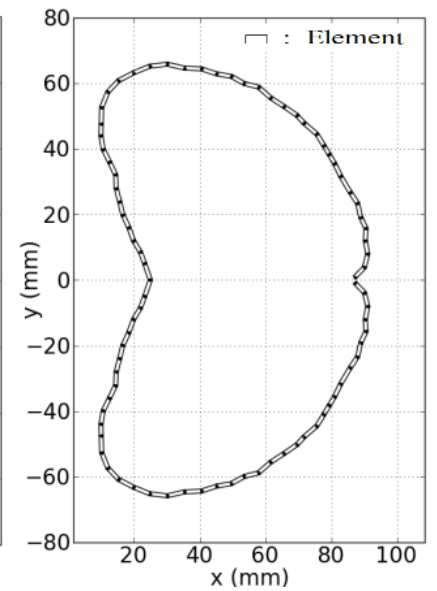

(d) $A_{s}=436.4 \mathrm{~mm}^{2}$ $N_{c}=74.94 \mathrm{kN}$ $N_{d} / A_{s}=171.7 \mathrm{MPa}$

Fig. 25: Optimised cross-sections for the 3,000 mm long columns and the non-manufacturable case, $(a, b)$ fittest cross-sections and (c, d) least fit cross-sections

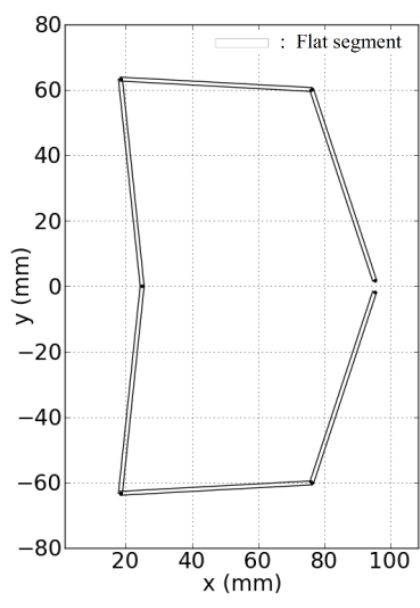

(a) $A_{s}=439.8 \mathrm{~mm}^{2}$ $N_{c}=75.17 \mathrm{kN}$ $N_{c} / A_{s}=170.9 \mathrm{MPa}$

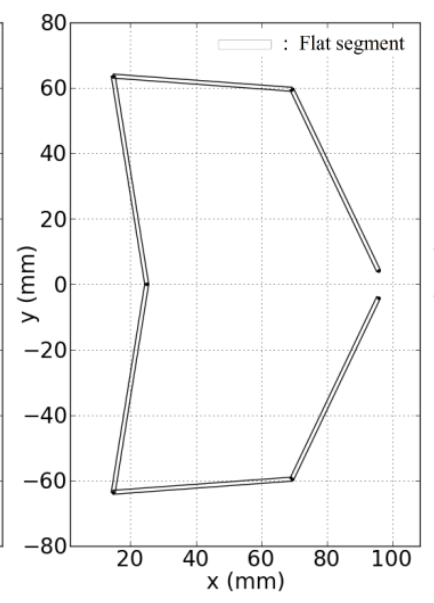

(b) $A_{s}=441.6 \mathrm{~mm}^{2}$ $N_{c}=75.31 \mathrm{kN}$ $N_{d} / A_{s}=170.5 \mathrm{MPa}$

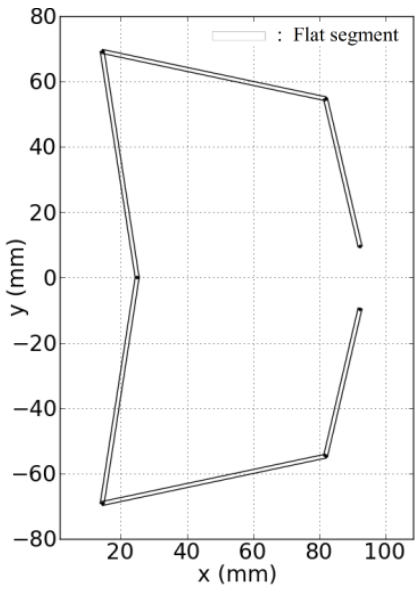

(c) $A_{s}=446.2 \mathrm{~mm}^{2}$ $N_{c}=75.24 \mathrm{kN}$ $N_{c} / A_{s}=168.6 \mathrm{MPa}$

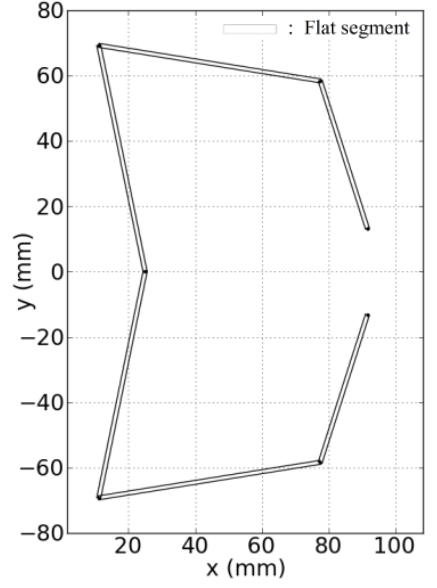

(d) $A_{s}=447.4 \mathrm{~mm}^{2}$ $N_{c}=75.30 \mathrm{kN}$ $N_{d} A_{s}=168.3 \mathrm{MPa}$

Fig. 26: Optimised cross-sections for the 3,000 mm long columns and $N_{\max }=3,(a, b)$ fittest crosssections and (c, d) least fit cross-sections 


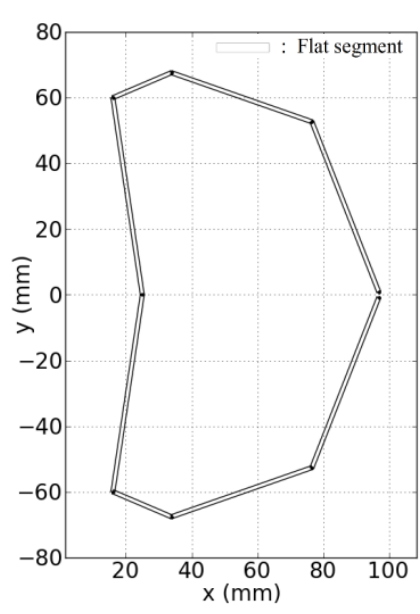

(a) $A_{s}=438.8 \mathrm{~mm}^{2}$
$N_{c}=75.57 \mathrm{kN}$
$N_{d} A_{s}=172.2 \mathrm{MPa}$

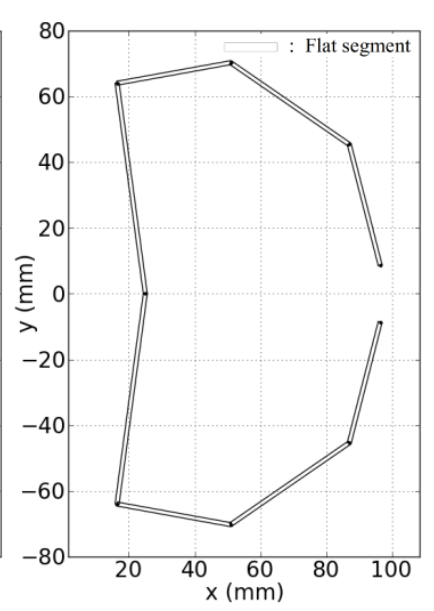

(b) $A_{s}=439.0 \mathrm{~mm}^{2}$ $N_{c}=75.23 \mathrm{kN}$

$N_{d} A_{s}=171.4 \mathrm{MPa}$
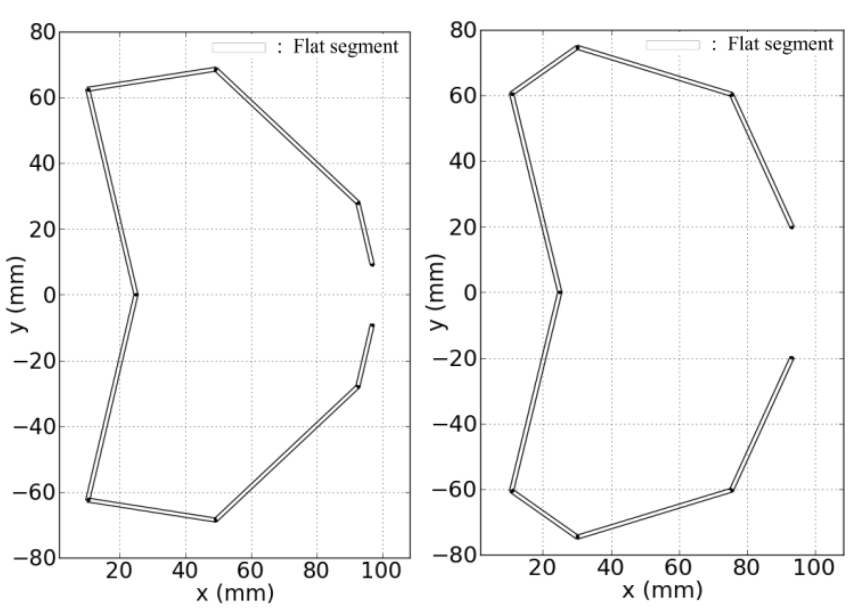

(c) $A_{s}=442.8 \mathrm{~mm}^{2}$ $N_{c}=75.38 \mathrm{kN}$

$N_{d} A_{s}=170.2 \mathrm{MPa}$ (d) $A_{s}=442.0 \mathrm{~mm}^{2}$ $N_{c}=75.19 \mathrm{kN}$ $N_{d} A_{s}=170.1 \mathrm{MPa}$

Fig. 27: Optimised cross-sections for the $3,000 \mathrm{~mm}$ long columns and $N_{\max }=4,(\mathrm{a}, \mathrm{b})$ fittest crosssections and (c, d) least fit cross-sections

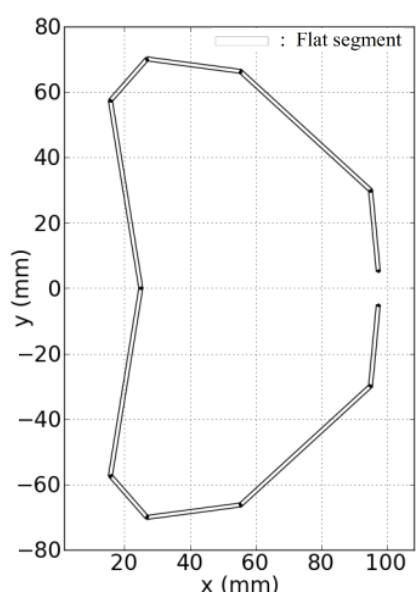

(a) $A_{s}=437.8 \mathrm{~mm}^{2}$ $N_{c}=75.78 \mathrm{kN}$ $N_{d} / A_{s}=173.1 \mathrm{MPa}$

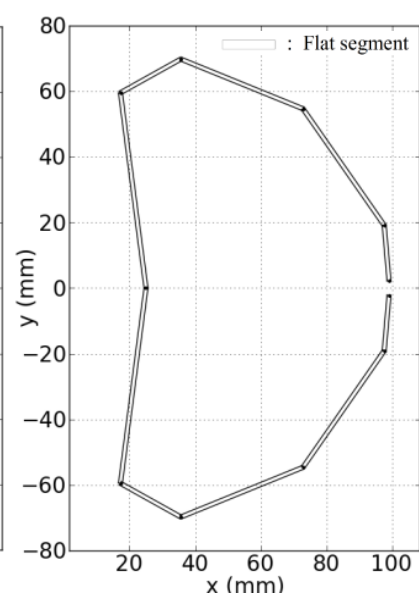

(b) $A_{s}=435.7 \mathrm{~mm}^{2}$ $N_{c}=75.22 \mathrm{kN}$ $N_{d} A_{s}=172.6 \mathrm{MPa}$

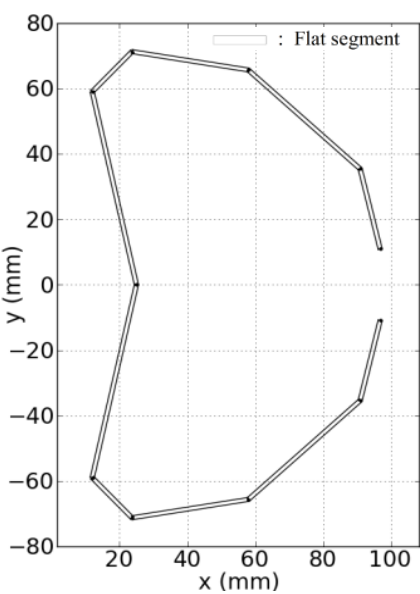

(c) $A_{s}=436.9 \mathrm{~mm}^{2}$ $N_{c}=75.04 \mathrm{kN}$ $N_{d} A_{s}=171.8 \mathrm{MPa}$

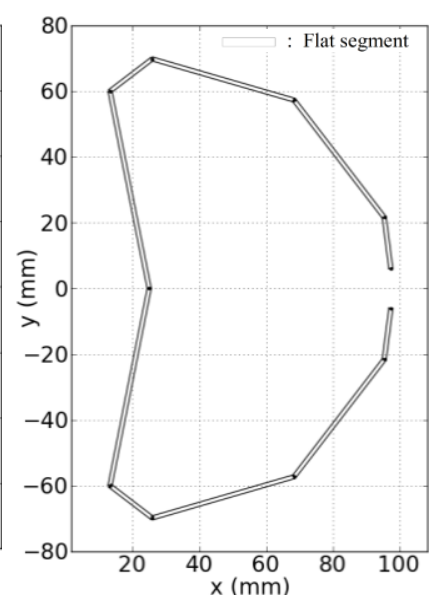

(d) $A_{s}=436.7 \mathrm{~mm}^{2}$ $N_{c}=75.01 \mathrm{kN}$ $N_{d} / A_{s}=171.8 \mathrm{MPa}$

Fig. 28: Optimised cross-sections for the $3,000 \mathrm{~mm}$ long columns and $N_{\max }=5,(\mathrm{a}, \mathrm{b})$ fittest crosssections and (c, d) least fit cross-sections 


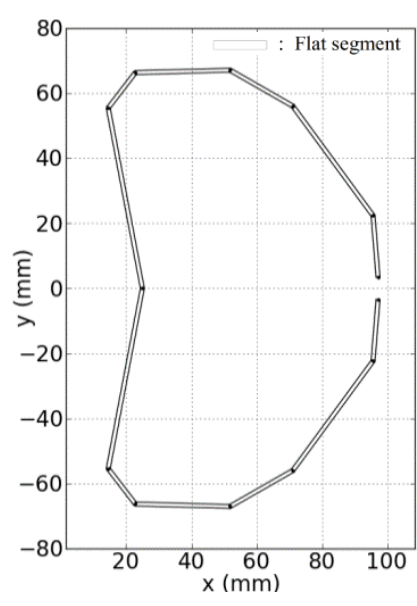

(a) $A_{s}=436.3 \mathrm{~mm}^{2}$ $N_{c}=75.75 \mathrm{kN}$

$N_{d} A_{s}=173.6 \mathrm{MPa}$

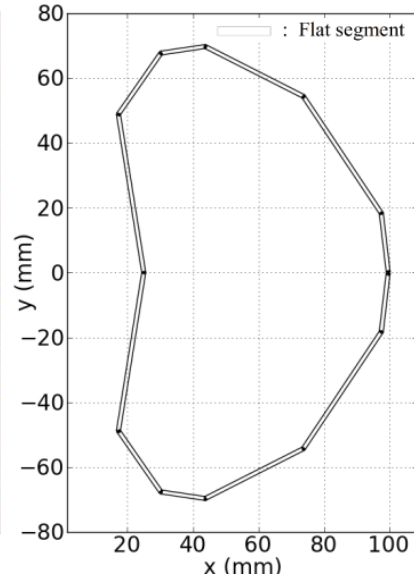

(b) $A_{s}=434.3 \mathrm{~mm}^{2}$ $N_{c}=75.11 \mathrm{kN}$ $N_{d} / A_{s}=172.9 \mathrm{MPa}$

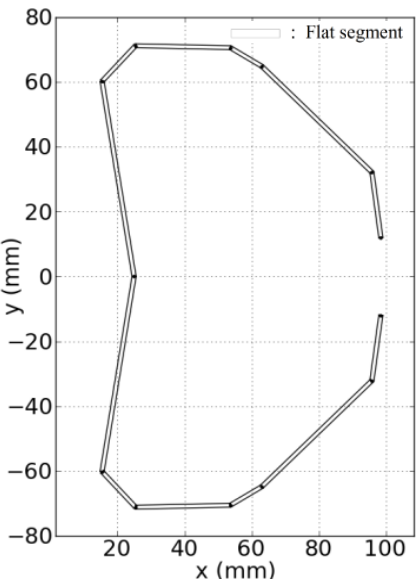

(c) $A_{s}=436.0 \mathrm{~mm}^{2}$ $N_{c}=74.81 \mathrm{kN}$ $N_{d} A_{s}=171.6 \mathrm{MPa}$

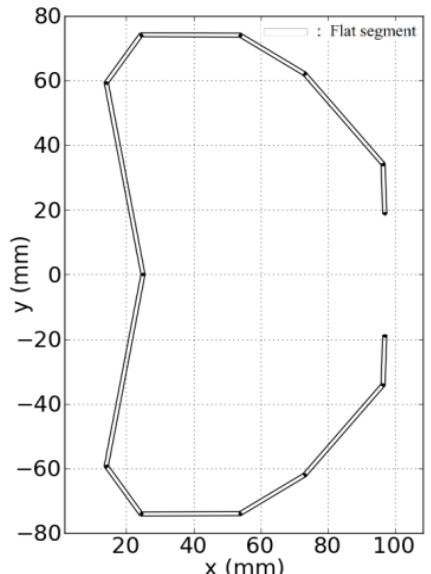

(d) $A_{s}=437.4 \mathrm{~mm}^{2}$ $N_{c}=74.80 \mathrm{kN}$ $N_{d} A_{s}=171.0 \mathrm{MPa}$

Fig. 29: Optimised cross-sections for the $3,000 \mathrm{~mm}$ long columns and $N_{\max }=6,(\mathrm{a}, \mathrm{b})$ fittest crosssections and (c, d) least fit cross-sections
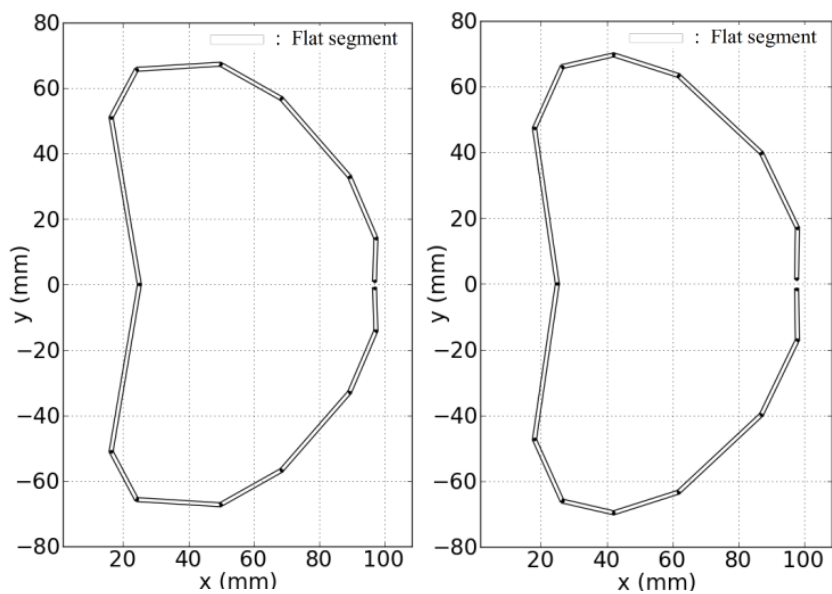
(a) $A_{s}=433.9 \mathrm{~mm}^{2}$ $N_{c}=75.17 \mathrm{kN}$ $N_{d} / A_{s}=173.2 \mathrm{MPa}$

(b) $A_{s}=433.0 \mathrm{~mm}^{2}$ $N_{c}=74.90 \mathrm{kN}$ $N_{d} / A_{s}=172.9 \mathrm{MPa}$

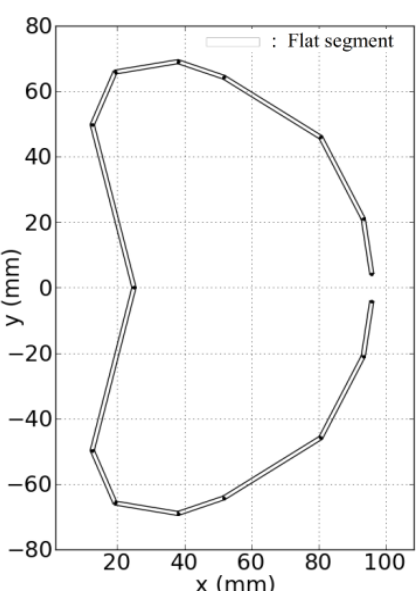

(c) $A_{s}=434.5 \mathrm{~mm}^{2}$ $N_{c}=74.98 \mathrm{kN}$ $N_{d} A_{s}=172.6 \mathrm{MPa}$

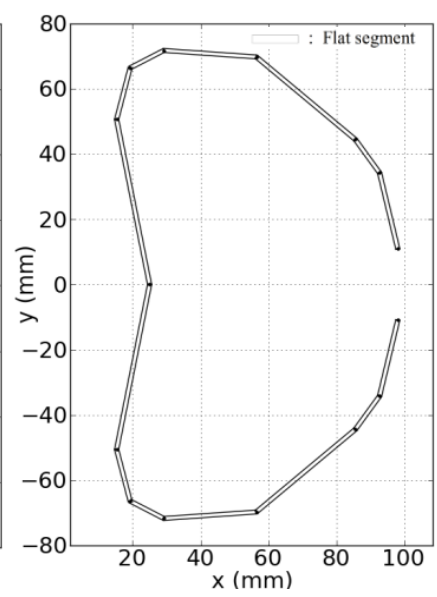

(d) $A_{s}=435.4 \mathrm{~mm}^{2}$ $N_{c}=75.07 \mathrm{kN}$ $N_{d} A_{s}=172.4 \mathrm{MPa}$

Fig. 30: Optimised cross-sections for the $3,000 \mathrm{~mm}$ long columns and $N_{\max }=7,(\mathrm{a}, \mathrm{b})$ fittest crosssections and (c, d) least fit cross-sections 


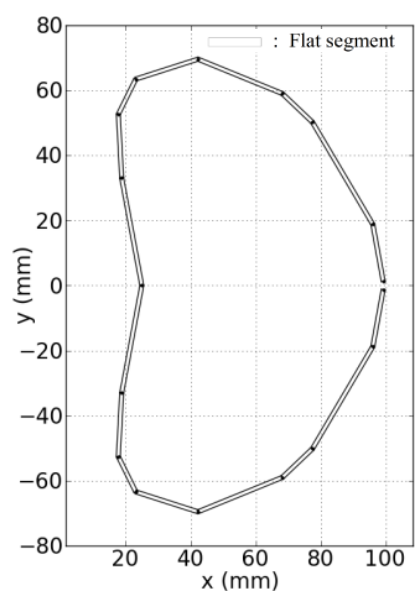

(a) $A_{s}=433.1 \mathrm{~mm}^{2}$ $N_{c}=74.99 \mathrm{kN}$

$N_{d} A_{s}=173.2 \mathrm{MPa}$

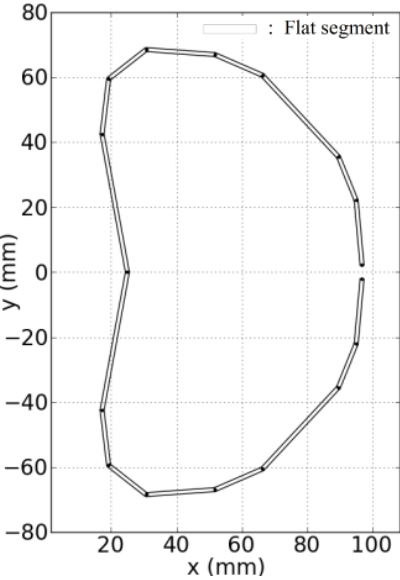

(b) $A_{s}=433.3 \mathrm{~mm}^{2}$ $N_{c}=75.00 \mathrm{kN}$ $N_{d} / A_{s}=173.1 \mathrm{MPa}$

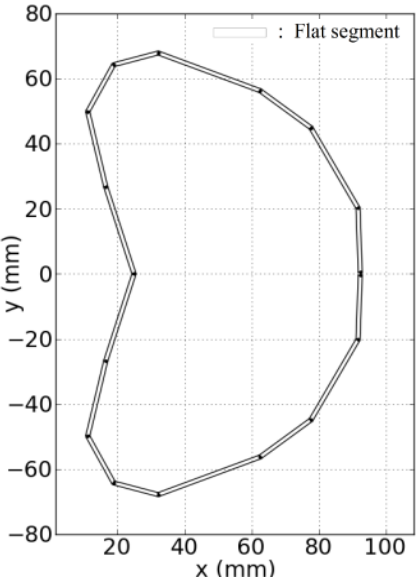

(c) $A_{s}=435.4 \mathrm{~mm}^{2}$ $N_{c}=75.02 \mathrm{kN}$ $N_{d} A_{s}=172.3 \mathrm{MPa}$

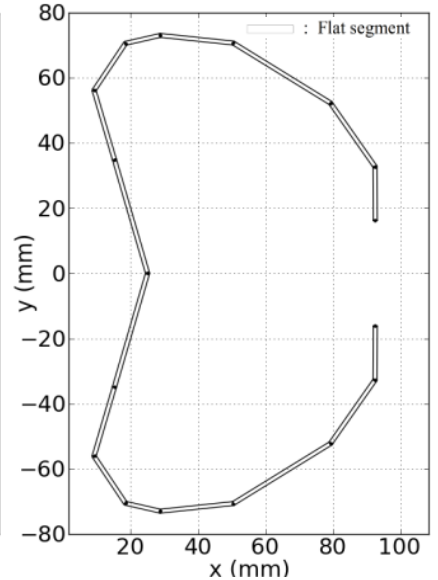

(d) $A_{s}=437.3 \mathrm{~mm}^{2}$ $N_{c}=74.93 \mathrm{kN}$ $N_{d} / A_{s}=171.4 \mathrm{MPa}$

Fig. 31: Optimised cross-sections for the $3,000 \mathrm{~mm}$ long columns and $N_{\max }=8,(\mathrm{a}, \mathrm{b})$ fittest crosssections and (c, d) least fit cross-sections 


\section{TABLES}

Table 1. AL penalty function coefficient $\gamma_{\text {align }}$ and associated weight $\omega$ selected for parametric study

\begin{tabular}{c|c|c|c|c}
\hline \multirow{2}{*}{ Combination } & \multicolumn{3}{|c}{ Valign } \\
\cline { 3 - 5 } \multicolumn{2}{c|}{} & 0.01 & 0.10 & 1.00 \\
\hline \multirow{3}{*}{$\omega$} & 0.3 & $(1)$ & $(4)$ & $(7)$ \\
\cline { 2 - 5 } & 0.5 & $(2)$ & $(5)$ & $(8)$ \\
\cline { 2 - 5 } & 0.7 & $(3)$ & $(6)$ & $(9)$ \\
\hline
\end{tabular}

Table 2. Average results over 10 runs for parametric study of $Y$ align and $\omega$

\begin{tabular}{c|c|c|c|c|c|c|c|c|c}
\hline \multirow{2}{*}{ Combination } & \multicolumn{3}{|c|}{ Cross-sectional area } & \multicolumn{2}{c|}{$\begin{array}{c}\text { Second moment } \\
\text { of area } I_{x}\end{array}$} & \multicolumn{2}{c|}{$\begin{array}{c}\text { Second moment } \\
\text { of area } I_{y}\end{array}$} & \multicolumn{2}{c}{$\begin{array}{c}\text { nbAligned/ } \\
\text { nbElement }\end{array}$} \\
\cline { 2 - 11 } & $\begin{array}{c}A_{s} \\
\left(\mathrm{~mm}^{2}\right)\end{array}$ & $\begin{array}{c}\text { Error } \\
(\%)\end{array}$ & $\begin{array}{c}\text { CoV } \\
(\%)\end{array}$ & $\begin{array}{c}\text { Error }\left({ }^{(2)}\right. \\
(\%)\end{array}$ & $\begin{array}{c}\text { CoV } \\
(\%)\end{array}$ & $\begin{array}{c}\text { Error }(2) \\
(\%)\end{array}$ & $\begin{array}{c}\text { CoV } \\
(\%)\end{array}$ & $\begin{array}{c}\text { Error } \\
(\%)\end{array}$ & $\begin{array}{c}\text { CoV } \\
(\%)\end{array}$ \\
\hline$(1)$ & 132.01 & -0.4 & 0.5 & -0.1 & 3.7 & -0.2 & 2.1 & -1.1 & 3.4 \\
\hline$(2)$ & 132.32 & -0.2 & 0.6 & 0.5 & 2.3 & -0.3 & 1.6 & 0.0 & 0.0 \\
\hline$(3)$ & 132.36 & -0.1 & 0.6 & 0.5 & 2.0 & 0.4 & 0.8 & 0.0 & 0.0 \\
\hline$(4)$ & 132.13 & -0.3 & 0.4 & 0.1 & 0.6 & 0.4 & 0.6 & 0.0 & 0.0 \\
\hline$(5)$ & 131.96 & -0.4 & 0.2 & 0.1 & 0.2 & 0.1 & 0.3 & 0.0 & 0.0 \\
\hline$(6)$ & 132.20 & -0.3 & 0.3 & 0.0 & 0.2 & 0.4 & 0.6 & 0.0 & 0.0 \\
\hline$(7)$ & 132.88 & 0.2 & 0.3 & 0.2 & 0.7 & 0.6 & 1.0 & 0.0 & 0.0 \\
\hline$(8)$ & 133.12 & 0.4 & 0.9 & 1.4 & 4.0 & 0.2 & 0.5 & 0.0 & 0.0 \\
\hline$(9)$ & 133.71 & 0.9 & 0.6 & 1.0 & 2.5 & 0.6 & 1.1 & 0.0 & 0.0 \\
\hline
\end{tabular}

Note: the negative error represents the optimised results are less than the optimum octagon's

(1): Relative error when compared to $A_{o}=132.55 \mathrm{~mm}^{2}$ of the optimum octagon

(2): Relative error when compared to $I_{x t}=I_{y t}$ are $28043.3 \mathrm{~mm}^{4}$ of the optimum octagon

Table 3. Alignment tolerance $\Delta r$ selected for parametric study

\begin{tabular}{c|c}
\hline$\Delta r(\mathrm{~mm})$ & $\Delta r / L_{\text {ele }}$ \\
\hline $0.5 t$ & 0.03 \\
\hline $1.0 t$ & 0.06 \\
\hline $1.5 t$ & 0.09 \\
\hline $2.0 t$ & 0.12 \\
\hline
\end{tabular}

Table 4. Average results over 10 runs for parametric study of $\Delta r$

\begin{tabular}{c|c|c|c|c|c|c|c|c|c}
\hline \multirow{2}{*}{$\begin{array}{c}\text { Case } \\
\text { of } \Delta r\end{array}$} & \multicolumn{3}{|c|}{ Cross-sectional area } & \multicolumn{2}{c|}{$\begin{array}{c}\text { Second moment } \\
\text { of area } I_{x}\end{array}$} & \multicolumn{2}{c|}{$\begin{array}{c}\text { Second moment } \\
\text { of area } I_{y}\end{array}$} & \multicolumn{2}{|c|}{$\begin{array}{c}\text { nbAligned/ } \\
\text { nbElement }\end{array}$} \\
\cline { 2 - 10 } & $\begin{array}{c}A_{s} \\
\left(\mathrm{~mm}^{2}\right)\end{array}$ & $\begin{array}{c}\text { Error } \\
(\%)\end{array}$ & $\begin{array}{c}\text { CoV } \\
(\%)\end{array}$ & $\begin{array}{c}\text { Error }(2) \\
(\%)\end{array}$ & $\begin{array}{c}\text { CoV } \\
(\%)\end{array}$ & $\begin{array}{c}\text { Error(2) } \\
(\%)\end{array}$ & $\begin{array}{c}\text { CoV } \\
(\%)\end{array}$ & $\begin{array}{c}\text { Error } \\
(\%)\end{array}$ & $\begin{array}{c}\text { CoV } \\
(\%)\end{array}$ \\
\hline $0.5 t$ & 131.96 & -0.4 & 0.2 & 0.1 & 0.2 & 0.1 & 0.3 & 0.0 & 0.0 \\
\hline $1.0 t$ & 131.06 & -1.1 & 0.1 & -0.1 & 0.2 & 0.1 & 0.1 & 0.0 & 0.0 \\
\hline $1.5 t$ & 131.23 & -1.0 & 0.1 & 0.2 & 0.4 & 0.1 & 0.3 & 0.0 & 0.0 \\
\hline $2.0 t$ & 130.43 & -1.6 & 0.0 & 0.0 & 0.1 & 0.0 & 0.1 & 0.0 & 0.0 \\
\hline
\end{tabular}

Note: the negative error represents the optimised results are less than the optimum octagon's

(1): Relative error when compared to $A_{o}=132.55 \mathrm{~mm}^{2}$ of the optimum octagon

(2): Relative error when compared to $I_{x t}=I_{y t}$ are $28043.3 \mathrm{~mm}^{4}$ of the optimum octagon 
Table 5: Alignment tolerance $\Delta r$ proposed for various $N_{\max }$ and column length $(t=1.2 \mathrm{~mm})$

\begin{tabular}{c|c|c|c|c|c|c|c|c|c|c|c}
\hline \multicolumn{4}{c|}{$500 \mathrm{~mm}$ Column } & \multicolumn{4}{c|}{$1,500 \mathrm{~mm}$ Column } & \multicolumn{3}{|c}{$3,000 \mathrm{~mm}$ Column } \\
\hline$N_{\max }$ & $\begin{array}{c}\Delta r \\
(\mathrm{~mm})\end{array}$ & $\begin{array}{c}L_{\max } \\
(\mathrm{mm})\end{array}$ & $\Delta r / L_{\max }$ & $N_{\max }$ & $\begin{array}{c}\Delta r \\
(\mathrm{~mm})\end{array}$ & $\begin{array}{c}L_{\max } \\
(\mathrm{mm})\end{array}$ & $\Delta r / L_{\max }$ & $N_{\max }$ & $\begin{array}{c}\Delta r \\
(\mathrm{~mm})\end{array}$ & $\begin{array}{c}L_{\max } \\
(\mathrm{mm})\end{array}$ & $\Delta r / L_{\max }$ \\
\hline 3 & $1.5 t$ & 40 & 0.045 & 3 & $2.5 t$ & 50 & 0.060 & 3 & $4.0 t$ & 65 & 0.074 \\
\hline 4 & $1.3 t$ & 32 & 0.049 & 4 & $2.5 t$ & 44 & 0.068 & 4 & $3.7 t$ & 60 & 0.074 \\
\hline 5 & $1.0 t$ & 32 & 0.038 & 5 & $2.0 t$ & 36 & 0.067 & 5 & $3.5 t$ & 46 & 0.091 \\
\hline 6 & $1.0 t$ & 32 & 0.038 & 6 & $1.5 t$ & 36 & 0.050 & 6 & $3.5 t$ & 46 & 0.091 \\
\hline- & - & - & - & 7 & $1.5 t$ & 36 & 0.050 & 7 & $3.0 t$ & 40 & 0.090 \\
\hline- & - & - & - & - & - & - & - & 8 & $2.5 t$ & 36 & 0.083 \\
\hline
\end{tabular}

Table 6: Average results over 10 runs for the $500 \mathrm{~mm}$ long columns

\begin{tabular}{c|c|c|c|c|c|c|c|c}
\hline \multirow{2}{*}{$N_{\max }$} & \multicolumn{2}{|c|}{$\begin{array}{c}\text { Cross-sectional } \\
\text { area }\end{array}$} & \multicolumn{3}{c|}{ Nominal member capacity } & $\begin{array}{c}\text { Ultimate } \\
\text { compressive } \\
\text { stress }\end{array}$ & \multicolumn{2}{c}{ Alignment } \\
\cline { 2 - 9 } & $\begin{array}{c}A_{s} \\
\left(\mathrm{~mm}^{2}\right)\end{array}$ & $\begin{array}{c}\text { CoV } \\
(\%)\end{array}$ & $\begin{array}{c}N_{c} \\
(\mathrm{kN})\end{array}$ & $\begin{array}{c}\text { Error(2) } \\
(\%)\end{array}$ & $\begin{array}{c}\text { CoV } \\
(\%)\end{array}$ & $\begin{array}{c}N_{c} / A_{s} \\
(\mathrm{MPa})\end{array}$ & $\begin{array}{c}\text { Error } \\
(\%)\end{array}$ & $\begin{array}{c}\text { CoV } \\
(\%)\end{array}$ \\
\hline 3 & 196.8 & 0.64 & 74.99 & 0.06 & 0.08 & 381.0 & 0.00 & 0.00 \\
\hline 4 & 195.4 & 0.33 & 75.01 & 0.04 & 0.05 & 383.9 & 0.00 & 0.00 \\
\hline 5 & 196.1 & 0.63 & 74.98 & 0.05 & 0.07 & 382.4 & 0.00 & 0.00 \\
\hline 6 & 195.6 & 0.74 & 75.00 & 0.03 & 0.04 & 383.4 & 0.00 & 0.00 \\
\hline$\infty(1)$ & 194.7 & 0.48 & 75.00 & 0.16 & 0.19 & 385.2 & -- & -- \\
\hline
\end{tabular}

(1): Algorithm ran without manufacturing constraints (non-manufacturable cross-section) (2): Absolute error when compared to $N^{*}=75 \mathrm{kN}$

Table 7: Average results over 10 runs for the $1,500 \mathrm{~mm}$ long columns

\begin{tabular}{|c|c|c|c|c|c|c|c|c|}
\hline \multirow{2}{*}{$N_{\max }$} & \multicolumn{2}{|c|}{$\begin{array}{c}\text { Cross-sectional } \\
\text { area }\end{array}$} & \multicolumn{3}{|c|}{ Nominal member capacity } & \multirow{2}{*}{$\begin{array}{c}\begin{array}{c}\text { Ultimate } \\
\text { compressive } \\
\text { stress }\end{array} \\
N_{c} / A_{s} \\
(\mathrm{MPa})\end{array}$} & \multicolumn{2}{|c|}{ Alignment } \\
\hline & $\begin{array}{c}A_{s} \\
\left(\mathrm{~mm}^{2}\right)\end{array}$ & $\begin{array}{l}\text { CoV } \\
(\%)\end{array}$ & $\begin{array}{c}N_{c} \\
(\mathrm{kN})\end{array}$ & $\begin{array}{c}\text { Error }^{(2)} \\
(\%)\end{array}$ & $\begin{array}{l}\text { CoV } \\
(\%)\end{array}$ & & $\begin{array}{c}\text { Error } \\
(\%)\end{array}$ & $\begin{array}{l}\text { CoV } \\
(\%)\end{array}$ \\
\hline 3 & 297.0 & 0.91 & 75.30 & 0.40 & 0.49 & 253.6 & 0.0 & 0.00 \\
\hline 4 & 292.1 & 0.56 & 74.97 & 0.23 & 0.29 & 256.7 & 0.0 & 0.00 \\
\hline 5 & 290.4 & 0.27 & 74.99 & 0.12 & 0.13 & 258.5 & 0.0 & 0.00 \\
\hline 6 & 289.6 & 0.35 & 75.07 & 0.06 & 0.06 & 259.0 & 0.0 & 0.00 \\
\hline 7 & 289.1 & 0.31 & 75.01 & 0.04 & 0.05 & 259.4 & 0.0 & 0.00 \\
\hline$\infty(1)$ & 288.1 & 0.26 & 74.97 & 0.16 & 0.20 & 260.2 & -- & -- \\
\hline
\end{tabular}

(1): Algorithm ran without manufacturing constraints (non-manufacturable cross-section) (2): Absolute error when compared to $N^{*}=75 \mathrm{kN}$ 
Table 8: Average results over 10 runs for the $3,000 \mathrm{~mm}$ long columns

\begin{tabular}{|c|c|c|c|c|c|c|c|c|}
\hline \multirow{2}{*}{$N_{\max }$} & \multicolumn{2}{|c|}{$\begin{array}{l}\text { Cross-sectional } \\
\text { area }\end{array}$} & \multicolumn{3}{|c|}{ Nominal member capacity } & \multirow{2}{*}{$\begin{array}{c}\begin{array}{c}\text { Ultimate } \\
\text { compressive } \\
\text { stress }\end{array} \\
N_{c} / A_{s} \\
(\mathrm{MPa})\end{array}$} & \multicolumn{2}{|c|}{ Alignment } \\
\hline & $\begin{array}{c}A_{s} \\
\left(\mathrm{~mm}^{2}\right)\end{array}$ & $\begin{array}{l}\text { CoV } \\
(\%)\end{array}$ & $\begin{array}{c}N_{c} \\
(\mathrm{kN})\end{array}$ & $\begin{array}{c}\text { Error (2) }^{(2)} \\
(\%)\end{array}$ & $\begin{array}{l}\mathrm{CoV} \\
(\%)\end{array}$ & & $\begin{array}{c}\text { Error } \\
(\%)\end{array}$ & $\begin{array}{l}\text { CoV } \\
(\%)\end{array}$ \\
\hline 3 & 444.7 & 0.64 & 75.37 & 0.50 & 0.35 & 169.5 & 0.0 & 0.00 \\
\hline 4 & 440.3 & 0.32 & 75.30 & 0.40 & 0.32 & 171.0 & 0.0 & 0.00 \\
\hline 5 & 436.5 & 0.20 & 75.15 & 0.20 & 0.30 & 172.2 & 0.0 & 0.00 \\
\hline 6 & 435.8 & 0.23 & 75.10 & 0.13 & 0.34 & 172.3 & 0.0 & 0.00 \\
\hline 7 & 434.3 & 0.19 & 75.03 & 0.04 & 0.16 & 172.8 & 0.0 & 0.00 \\
\hline 8 & 434.5 & 0.29 & 75.02 & 0.02 & 0.08 & 172.7 & 0.0 & 0.00 \\
\hline$\infty(1)$ & 435.3 & 0.35 & 75.16 & 0.21 & 0.54 & 172.7 & -- & -- \\
\hline
\end{tabular}

(1): Algorithm ran without manufacturing constraints (non-manufacturable cross-section) (2): Absolute error when compared to $N^{*}=75 \mathrm{kN}$

Table 9: Results of conventional lipped channel cross-sections

\begin{tabular}{c|c|c|c|c|c|c|c|c}
\hline \multirow{2}{*}{$\begin{array}{c}\text { Column } \\
\text { length } \\
(\mathrm{mm})\end{array}$} & $\begin{array}{c}\text { Depth } \\
(\mathrm{mm})\end{array}$ & $\begin{array}{c}\text { Width } \\
(\mathrm{mm})\end{array}$ & $\begin{array}{c}\text { Lip } \\
(\mathrm{mm})\end{array}$ & $\begin{array}{c}\text { Thick- } \\
\text { ness } \\
(\mathrm{mm})\end{array}$ & $\begin{array}{c}\text { Cross- } \\
\text { section } \\
\text { area } \\
\left(\mathrm{mm}^{2}\right)\end{array}$ & $\begin{array}{c}\text { Nominal } \\
\text { member } \\
\text { capacity } \\
(\mathrm{kN})\end{array}$ & $\begin{array}{c}\text { Ultimate } \\
\text { compressive } \\
\text { stress } \\
(\mathrm{MPa})\end{array}$ & $\begin{array}{c}\text { Improvement } \\
\text { in capacity } \\
(\%)\end{array}$ \\
\hline 500 & 68.6 & 42.3 & 10.8 & 1.2 & 196.0 & 57.7 & 294.4 & 30 \\
\hline 1,500 & 118.6 & 55.3 & 13.1 & 1.2 & 290.3 & 39.3 & 135.4 & 91 \\
\hline 3,000 & 195.2 & 75.2 & 16.5 & 1.2 & 435.3 & 29.9 & 68.7 & 151 \\
\hline
\end{tabular}

(1): Equal to the average optimised cross-sectional area of manufacturable cases (2): Compared to the average optimised capacity of manufacturable cases 